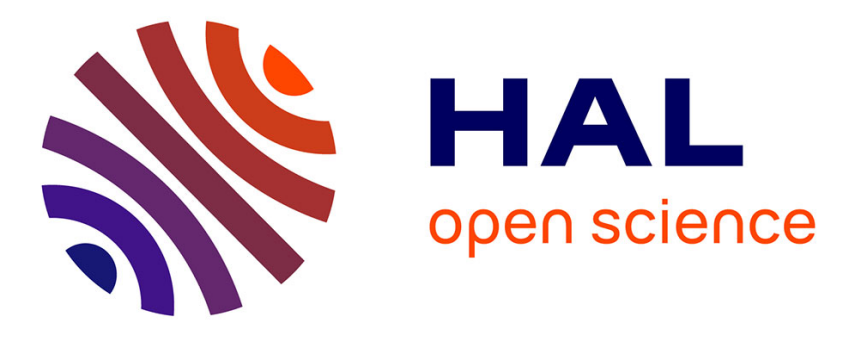

\title{
Design and security analysis of two robust keyed hash functions based on chaotic neural networks
}

Nabil Abdoun, Safwan El Assad, Olivier Déforges, Rima Assaf, Mohamad Khalil

\section{- To cite this version:}

Nabil Abdoun, Safwan El Assad, Olivier Déforges, Rima Assaf, Mohamad Khalil. Design and security analysis of two robust keyed hash functions based on chaotic neural networks. Journal of Ambient Intelligence and Humanized Computing, 2020, 11 (5), pp.2137-2161. 10.1007/s12652-019-01244-y . hal-02048419

\section{HAL Id: hal-02048419 \\ https://hal.science/hal-02048419}

Submitted on 8 Mar 2019

HAL is a multi-disciplinary open access archive for the deposit and dissemination of scientific research documents, whether they are published or not. The documents may come from teaching and research institutions in France or abroad, or from public or private research centers.
L'archive ouverte pluridisciplinaire HAL, est destinée au dépôt et à la diffusion de documents scientifiques de niveau recherche, publiés ou non, émanant des établissements d'enseignement et de recherche français ou étrangers, des laboratoires publics ou privés. 


\title{
Design and security analysis of two robust keyed hash functions based on chaotic neural networks
}

\author{
Nabil Abdoun · Safwan El Assad • Olivier \\ Deforges · Rima Assaf • Mohamad Khalil
}

\begin{abstract}
In this paper, we designed, implemented, and analyzed the performance, in terms of security and speed, of two proposed keyed Chaotic Neural Network $(C N N)$ hash functions based on Merkle-Dåmgard $(M D)$ construction with three output schemes: CNN-Matyas-Meyer-Oseas, Modified CNN-Matyas-MeyerOseas, and CNN-Miyaguchi-Preneel. The first hash function's structure is composed of two-layer chaotic neural network while the structure of the second hash function is formed of one-layer chaotic neural network followed by non-linear layer functions. The obtained results of several statistical tests and cryptanalytic analysis highlight the robustness of the proposed keyed $C N N$ hash functions, which is fundamentally due to the strong non-linearity of both the chaotic systems and the neural networks. The comparison of the performance analysis with some chaosbased hash functions of the literature and with standard hash functions make the proposed hash functions suitable for data integrity, message authentication, and digital signature applications.
\end{abstract}

Nabil Abdoun - corresponding author

Institut d'Électronique et de Télécommunications de Rennes, UMR CNRS 6164 / site Polytech Nantes

Tel.: +33669417198

E-mail: nabil.abdoun@etu.univ-nantes.fr

Safwan El Assad

Institut d'Électronique et de Télécommunications de Rennes, UMR CNRS 6164 / site Polytech Nantes

Tel.: +33676322836

E-mail: safwan.elassad@univ-nantes.fr

Olivier Deforges

Institut d'Électronique et de Télécommunications de Rennes / site INSA Rennes

E-mail: olivier.deforges@insa-rennes.fr

Rima Assaf

Lebanese University

E-mail: rima.assaf@ul.edu.lb

Mohamad Khalil

Lebanese University

E-mail: mohamad.khalil@ul.edu.lb 
Keywords Keyed hash functions · Chaotic Neural Networks · Chaotic activation function · Merkle-Dåmgard · Statistical tests · Brute force attacks · Cryptanalytical attacks $\cdot$ Speed analysis

\section{Introduction}

During the last decade, information security has become a hot issue. Developers are usually concerned about five main services regarding information exchange over non-secure channels (e.g., Internet): confidentiality, authenticity, integrity, non-repudiate, and availability. Hash functions are one of the most useful primitives in cryptography that play an important role in data security. They can achieve data integrity, message authentication [1], and digital signature [2]. Hash function is a one-way function that maps an arbitrary finite large message data into a fixed-length hash value. It should achieve some security properties, such as message sensitivity, key sensitivity, confusion-diffusion, preimage, second preimage, and collision resistance. Also, it should be immune against brute force and cryptanalytical attacks. Nowadays, the most popular standard secure hash functions are unkeyed Secure Hash Algorithms $S H A-2$ [3] and $S H A-3$ [4], commonly used by many $S S L$ certificate authorities, whereas keyed hash functions include: Very fast Message Authentication Code-VMAC, Keyed-Hash MAC-HMAC, Galios / Counter Mode-GCM, Cipher-based MAC-CMAC, Destination MAC-DMAC, Cipher Block Chaining Message Authentication Code-CBC-MAC and BLAKE 2.

Alternatively, a new direction in the construction of chaos-based hash functions appeared in 2002. Due to the strong non-linearity of chaotic systems and neural network structures, some designers usually combine these two systems to build robust hash functions. Indeed, a chaotic system is characterized by important security features, such as sensitivity to initial conditions, random-like behavior, and unstable periodic orbits. Also, a neural network is characterized by its

confusion-diffusion and compression properties that are required to design secure hash functions.

However, many researchers developed hashing schemes based on simple chaotic maps, such as logistic map, high-dimensional discrete map, piecewise linear chaotic map, tent map, and Lorenz map or on 2D coupled map lattices [5-18]. In 2007, Zhang et al., [19] proposed a novel chaotic keyed hash algorithm using a feed forward-feedback nonlinear filter. Other researchers proposed combined hashing and encryption schemes based on chaotic neural network [20-32].

Since 2010, there has been a real turning point in building new secure hash algorithms based on chaotic maps and neural network. Huang [33] proposed an enhancement of Xiao's parallel keyed hash function based on chaotic neural network [24]. Indeed, in Xiao's scheme, the secret keys are not nonce numbers, which might produce a potential security flaw. Jiteurtragool et al. [34], proposed a topologically simple keyed hash function based on circular chaotic sinusoidal map network that uses more complex map, i.e., the Sine map. In 2014, Teh et al., [35] introduced a parallel chaotic hash function based on the shuffle-exchange network that runs in parallel to improve hashing speed. In 2015, Abdoun et al., [36, 37] proposed a new efficient structure that consists of two parts: an efficient chaotic generator and a three or two-layer neural network. Chenaghlu et al., [38] published a new keyed parallel hashing scheme based on a new hyper sensitive chaotic 
system with compression ability. High-dimensional chaotic maps have also been used in hash functions for higher complexity and better mixing [39-41]. Xiao et al., [42] designed a parallel keyed chaos-based hash function, where a mechanism of both changeable-parameter and self-synchronization is used to establish a close relation of the keystream with the algorithm key, the content, and the order of each message block.

This paper proposes two robust keyed $C N N$ hash functions based on MerkleDåmgard construction, that having better hash throughput as compared to the other chaos-based hash functions in literature. Indeed, the structures of the proposed $C N N$ hash functions are based on neural network layer(s) and non-linear layer functions. Each neuron uses a chaotic activation function based on an efficient chaotic generator using Discrete Skew Tent map (DSTmap) and a Discrete Piecewise Linear Chaotic map (DPWLCmap) [43,44].

The rest of this paper is organized as follows: Sect. 2 presents the generalities, properties, and classification of cryptographic hash functions. The section also introduces the general model of Merkle-Dåmgard construction formed by preprocessing and compression phases. Sect. 3 introduces in detail the structures of the two proposed keyed $C N N$ hash functions based on $M D$ with their components i.e., chaotic generator, output schemes, neural network, and non-linear functions. Sect. 4 presents the obtained results, in terms of security and computational performance, of the proposed hash functions and compares their performance with other hash functions found in literature. Sect. 5 concludes our contribution and outlines the direction of future work.

\section{Preliminaries}

2.1 Generalities of cryptographic hash functions

Cryptographic hash functions play a fundamental role in modern cryptography. The basic idea of cryptographic hash functions is that a hash-value $h$ serves as a compact representative image (sometimes called an imprint, digital fingerprint, or message digest) of an input message $M$ and is used as an uniquely identifiable element (Fig. 1) [45-49]. Precisely, a cryptographic hash function $H$, that requires to be a deterministic process, maps bit-strings of arbitrary finite length $|M|$ to strings of fixed length ( $u$ bits), where $|M|>u$. So, every time if the same input message $M$ is hashed by $H$, the same hash value $h$ is obtained. $H$ is many-to-one relationship that implies the existence of unavoidable collisions (pairs of input message with identical output hash value) with very small probabilities.

A cryptographic hash function $H$ aims to guarantee a number of properties, which makes it very useful for information security. $H$ must verify at least the following two implementation properties:

1. Compression: $H$ maps an input message $M$ of arbitrary finite bit-length to a hash value $h$ of fixed bit-length $u$ bits.

2. Ease of computation: given $H$ and an input message $M, H(M)$ is easy to compute.

Nevertheless, two important requirements are needed to realize the cryptographic hash functions: the hardness to find collisions and the appearance of randomness. Also, $H$ has the following three security properties (Fig. 2): 


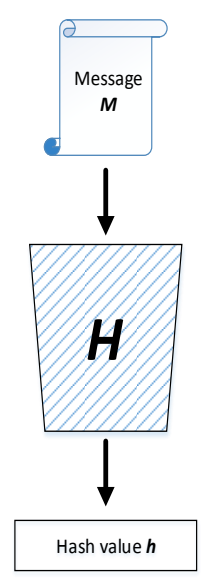

Fig. 1: Hash function

1. Preimage resistance (one-way): for all the pre-specified hash values $h$, it is computationally infeasible to find any message input that is hashed to the chosen hash value.

2. Second preimage resistance (weak collision resistance): it is computationally infeasible to find any second input that has the same hash value as a specified input message $M$.

3. Collision resistance (strong collision resistance): it is computationally infeasible to find any two distinct message inputs $\left(M, M^{\prime}\right)$ hashed to the same hash value, such that $H(M)=H\left(M^{\prime}\right)$. It should be noted that, the users are free to choose both input messages.

We should mention that the notion of computationally infeasible depends on the relationship between the amount of work the designer has to do to secure the system in comparison to the amount of work that the attacker has to do to break it. At the highest level, cryptographic hash functions are classified into two classes: Unkeyed and Keyed hash functions that are presented in Fig. 3. In this paper, our work is restricted to keyed cryptographic hash functions (simply called hash functions in the rest of this paper) that are originally proposed to generate the inputs of Digital Signature $(D S)$ application. Later, these hash functions are designed to achieve certain security properties, such as message authentication useful for building cryptosystems. In general, a keyed hash function [50] uses a secret key $K$. The Merkle-Dåmgard structure, which is unkeyed hash function that uses initial values $I V$, can be transformed to a keyed hash function by appending a secret key $K$ to the input message $M$ to produce the hash value $h$. Table 1 presents the two primary types of keyed hash functions $(M A C, D S)$ with their realized security goals and the kind of their used keys. 


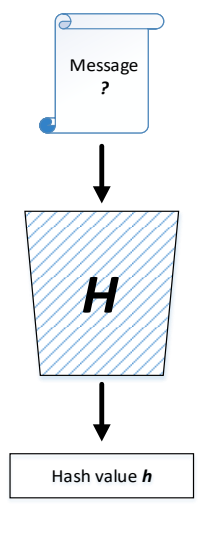

Preimage resistance

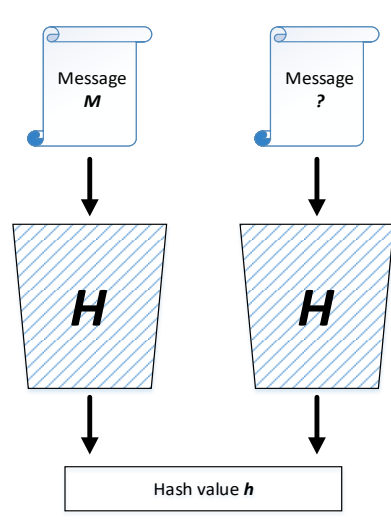

Second Preimage resistance

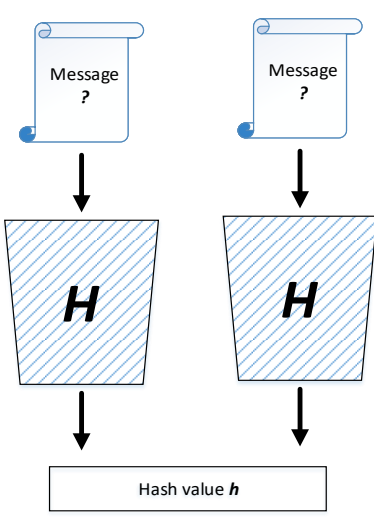

Collision resistance

Fig. 2: Security properties of hash functions

\begin{tabular}{ccc}
\hline Hash functions security goal & MAC & Digital Signature \\
\hline Integrity & Yes & Yes \\
Authentication & Yes & Yes \\
Non-repudiation & No & Yes \\
\hline Kind of keys & Symmetric keys & Asymmetric keys \\
\hline
\end{tabular}

Table 1: Two primary types of keyed hash functions

\subsection{Structures of hash functions}

In cryptography, many structures are used to construct different hash functions [51], such as Merkle-Dåmgard [52,53], Wide Pipe [54], Fast Wide Pipe [55], HAIFA [56], and Sponge construction [57]. The Merkle-Dåmgard construction was used in the design of many popular hash algorithms, such as MD5 [58], SHA-1 [59], and SHA-2 [3]. The Sponge construction was used in the design of $S H A-3$ [4]. This paper proposes novel hash functions based on Chaotic System and Neural Network. The proposal uses the structure of Merkle-Dåmgard with a proposed compression function based on Chaotic Neural Network $(C N N)$. To understand the proposed hash functions, it is necessary to introduce the Merkle-Daimgard construction (Fig.4) and the model of Strengthened Merkle-Dåmgard (Fig. 5).

Merkle-Dåmgard construction: preprocessing and compression : Fig. 4 shows the structure of Merkle-Dåmgard construction where the compression function is defined by $C:\{0,1\}^{l} \times\{0,1\}^{\left|M_{i}\right|} \rightarrow\{0,1\}^{l}$. $C$ takes as inputs a chaining or state variable $h_{i}(i=0, \ldots, q-1)$ of size $l$ bits and a message block $M_{i}(i=1, \ldots, q)$ of size $\left|M_{i}\right|$ bits, to produce the updated chaining variable $h_{i}(i=1, \ldots, q)$ of size $l$ bits. Thus, to allow the usage of input messages of arbitrary length, the MerkleDåmgard structure needs a padding, which transforms the input message into a padded message $M$ of length multiple of $\left|M_{i}\right|$ bits. Indeed, a simple padding is insufficient because, in this case, the generated hash value is vulnerable to different 


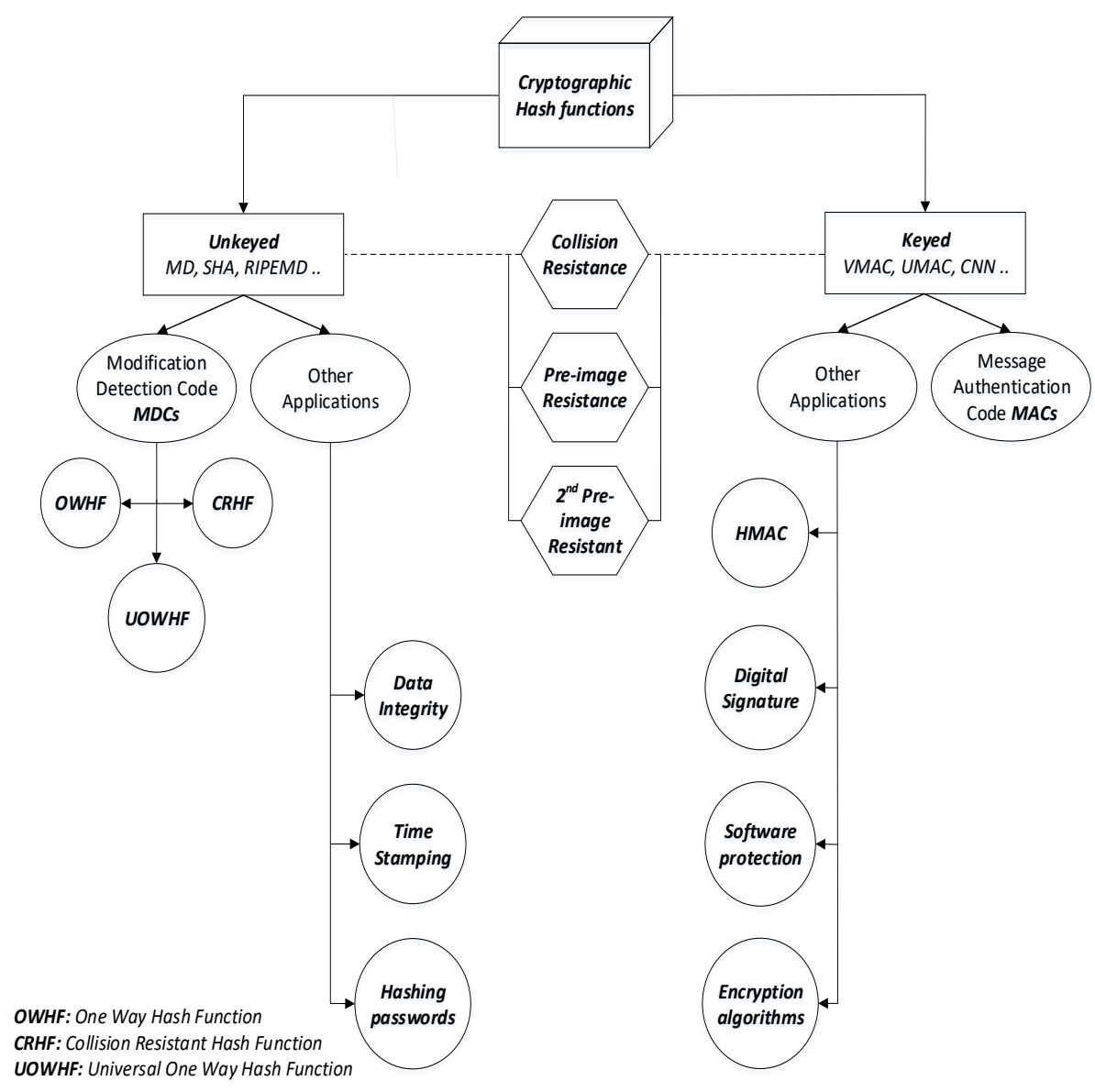

Fig. 3: Classification of cryptographic hash functions

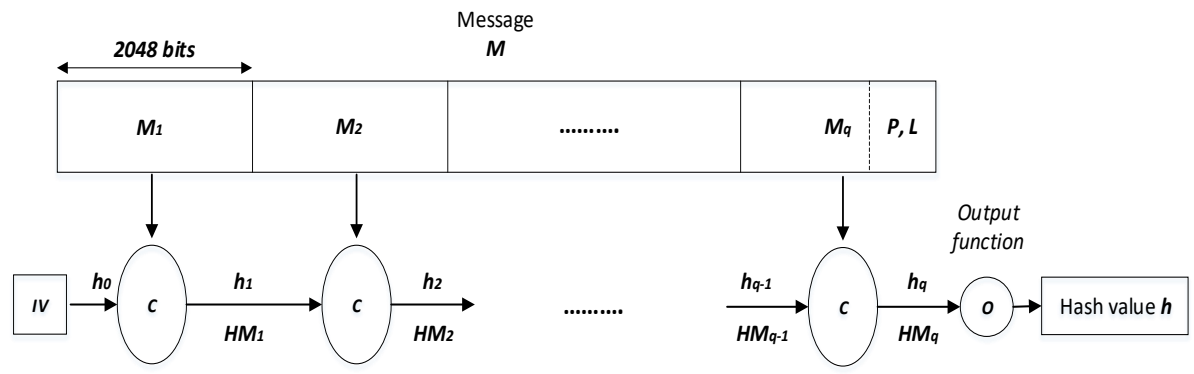

P: Padding, L: Length of the message M, C: Message compression function

Fig. 4: Strengthened Merkle-Dåmgard construction 


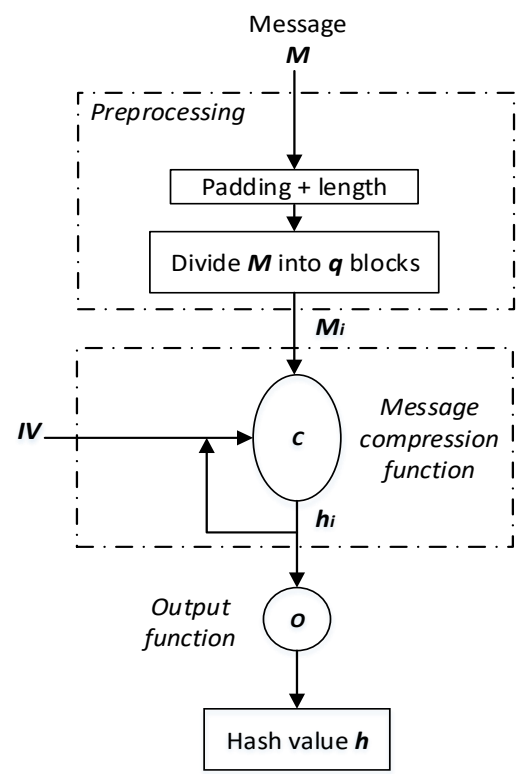

Fig. 5: Model of Strengthened Merkle-Dåmgard construction

attacks due to collision between the latest blocks. We will consider the Strengthened Merkle-Dåmgard padding with length strengthening (Figures 5 and 6). It uses a padding function named "is-pad", which appends the binary value of the message length $L$ at the end of the message to generate the padded message. Additionally, the Strengthened Merkle-Dåmgard construction employs a predefined initialization vector $I V$ used as the first state value of the structure. The Strengthened Merkle-Dåmgard hash function $S M D_{C}(M)$ is defined as follow:

$$
\begin{aligned}
& M_{1}\left\|M_{2}\right\| \ldots \| M_{q} \leftarrow \text { "is-pad }(M) " \\
& h_{0} \leftarrow I V \\
& \text { for } i=1, \ldots, q \text { do } h_{i} \leftarrow C\left(h_{i-1}, M_{i}\right) \\
& h \leftarrow O\left(h_{q}\right) \\
& \text { return } h .
\end{aligned}
$$

$M$ is padded with the bit pattern $00 \ldots 0$ of length $v$ bits, as shown in equation (1). The remaining 64 bits is used by "is-pad" function to denote $L$.

$$
v=\left|M_{i}\right|-\bmod \left[(L+64),\left|M_{i}\right|\right]
$$

It should be noted that, if $L$ exceeds $2^{64}$, then $L \bmod 2^{64}$ is taken as the message length instead of $L$ [46]. 

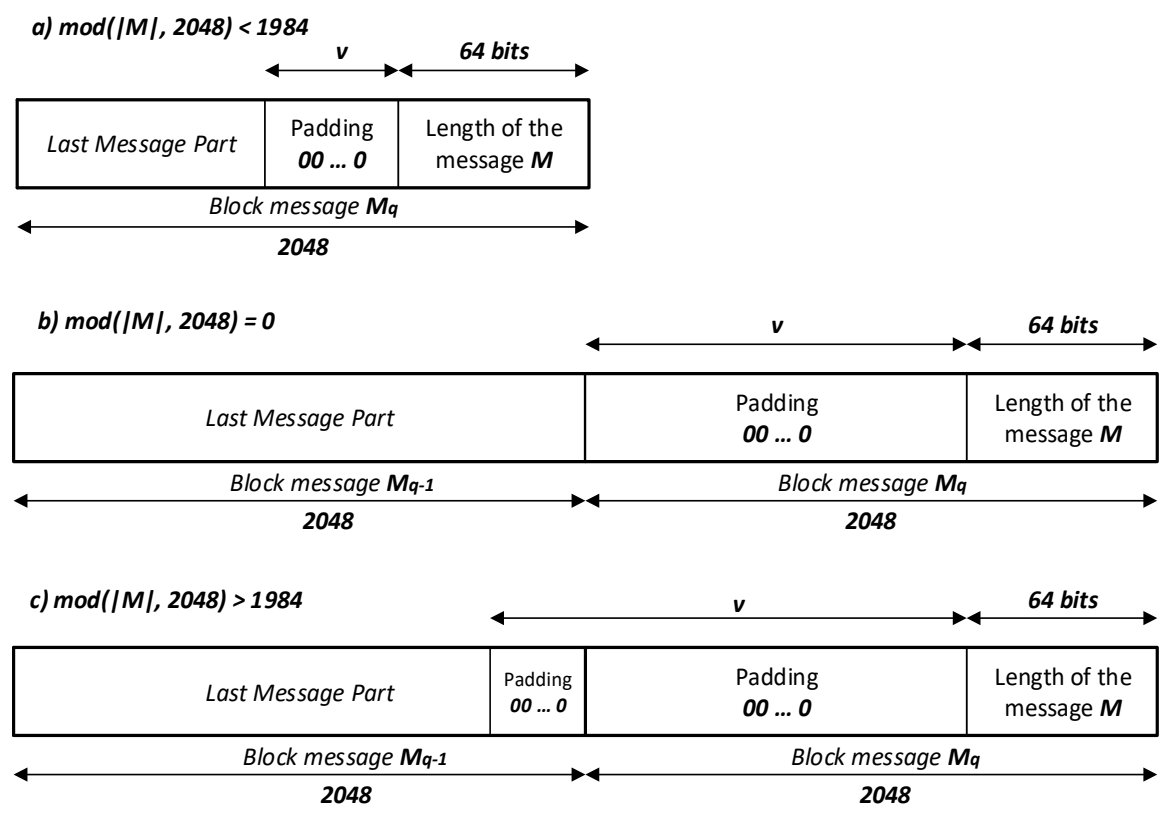

Fig. 6: The padding of input message in the proposed hash functions

In general, we have 3 cases of padding:

$$
\begin{aligned}
& \text { case } a: \bmod \left(|M|,\left|M_{i}\right|\right)<\left|M_{i}\right|-64 . \\
& \text { case } b: \bmod \left(|M|,\left|M_{i}\right|\right)=0 . \\
& \text { case } c: \bmod \left(|M|,\left|M_{i}\right|\right)>\left|M_{i}\right|-64 .
\end{aligned}
$$

Now, let's take a look at the three cases of padding where $\left|M_{i}\right|=2048$ bits (Fig. $6)$, which is as follows:

$$
\begin{aligned}
& \text { case } a: \text { if } L=6066 \text { bits }: \\
& v=2048-\bmod [(6066+64), 2048]=14 \text { bits. } \\
& \text { case } b: \text { if } L=6144 \text { bits }: \\
& v=2048-\bmod [(6144+64), 2048]=1984 \text { bits. } \\
& \text { case } c: \text { if } L=6086 \text { bits }: \\
& v=2048-\bmod [(6086+64), 2048]=2042 \text { bits. }
\end{aligned}
$$

Then, the padded message is processed as a sequence of message blocks $M_{1}\left\|M_{2}\right\|$ ... $\| M_{q}$. 


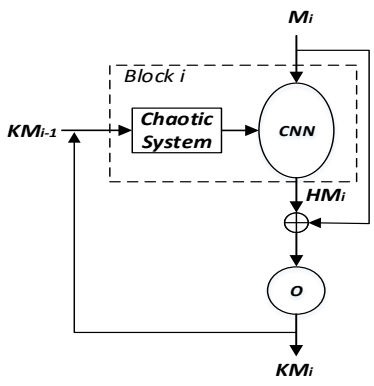

a) CNN-Matyas-Meyer-Oseas

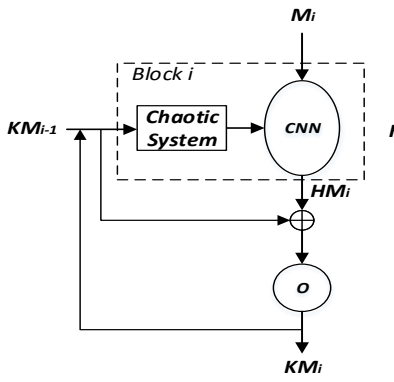

b) Modified CNN-Matyas-Meyer-Oseas

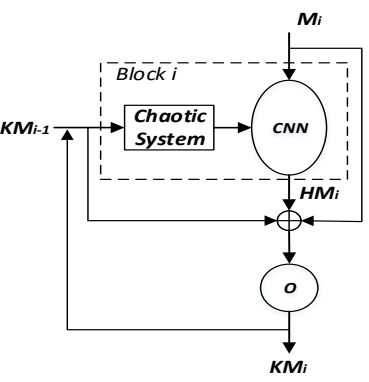

c) CNN-Miyaguchi-Preneel

Fig. 7: The proposed Merkle-Dåmgard compression functions based on $C N N$ with output schemes

\section{Chaotic Neural Network structure of the proposed keyed hash functions}

This paper proposes two keyed hash functions based on Chaotic Neural Network $(C N N)$, and for each one, three output schemes are suggested as presented in Fig. 7. The first $C N N$ hash function uses two-layer neural network structure (named Structure 1), whereas the second hash function uses one-layer neural network followed by a combination of Non-Linear $(N L)$ functions (named Structure 2). The next sub-section describes the three suggested output schemes based on MatyasMeyer-Oseas [60-62] and Miyaguchi-Preneel [63-66].

\subsection{Suggested output schemes}

Matyas-Meyer-Oseas (MMO) output scheme: In this output scheme, the message block $M_{i}$ is xored with the chaining variable $H M_{i}$, which is the output of the $C N N$ that takes as inputs $M_{i}$ and the output of the Chaotic System (Fig. 7-a). The state value $K M_{i-1}$ is the key of the Chaotic System. Due to the possible different bitlength, an output function $O$ precedes the generation of the final output $K M_{i}$, which represents the key of the next block, which is as follows:

$$
K M_{i}=O\left(H M_{i} \oplus M_{i}\right)
$$

where $i$ : the block index; $1 \leq i \leq q$.

for $i=1: K M_{0}=K$ : the secret key.

for $i=q: K M_{q}=h$ : the final hash value.

Modified Matyas-Meyer-Oseas (MMMO) output scheme: This output scheme is similar to $M M O$ output scheme except for the xor operation. Indeed in this case, $H M_{i}$ is xored with $K M_{i-1}$ (Fig. 7-b), where the final output $K M_{i}$ is defined by:

$$
K M_{i}=O\left(H M_{i} \oplus K M_{i-1}\right)
$$


where $i$ : the block index; $1 \leq i \leq q$.

for $i=1: K M_{0}=K$ : the secret key.

for $i=q: K M_{q}=h$ : the final hash value.

Miyaguchi-Preneel (MP) output scheme: This output scheme can be considered as an extension of the $M M O$ output scheme, where $K M_{i-1}$ is also added to the xor operation between $M_{i}$ and $H M_{i}$ (Fig. 7-c). The final output $K M_{i}$ is defined by:

$$
K M_{i}=O\left(H M_{i} \oplus M_{i} \oplus K M_{i-1}\right)
$$

where $i$ : the block index; $1 \leq i \leq q$.

for $i=1: K M_{0}=K$ : the secret key.

for $i=q: K M_{q}=h$ : the final hash value.

\subsection{Chaotic System}

The proposed Chaotic System is used to generate the parameters concerning the $C N N$ compression function (Fig. 7). It comprises the DSTmap with one recursive cell (delay equal to 1) (Fig. 8). Its outputs are defined as follows:

$$
\begin{aligned}
& K S s(n)=D S T m a p(K S s(n-1), Q 1) \\
& = \begin{cases}2^{N} \times \frac{K S s(n-1)}{Q 1} & \text { if } 0<K S s(n-1)<Q 1 \\
2^{N}-1 & \text { if } K S s(n-1)=Q 1 \\
2^{N} \times \frac{2^{N}-K S s(n-1)}{2^{N}-Q 1} & \text { if } Q 1<K S s(n-1)<2^{N}\end{cases}
\end{aligned}
$$

where $Q 1$, the control parameter, and $K S s(n)$ range from 1 to $2^{N}-1$. $\mathrm{N}$ is the finite precision and is equal to 32 bits. The secret key $K$, used for the first block $M_{1}$, is composed of the necessary parameters and initial conditions of the simplified version of the Chaotic Generator patent [43] and it is given by the following equation:

$$
K=\left\{K S s(0), K s, K S s(-1), U_{s}, Q 1\right\}
$$

where $K S s(0)$ and $K S s(0)$ are the initial values, $U_{s}$ is an additional initial value used only to generate the first sample, $K s$ is the coefficient, and $Q 1$ is the control parameter of the Chaotic System. The components of $K$ are samples of 32 bits length and its size is given as follows:

$$
\begin{aligned}
|K| & =|K S s(0)|+|K s|+|K S s(-1)|+\left|U_{s}\right|+|Q 1| \\
& =160 \text { bits }
\end{aligned}
$$




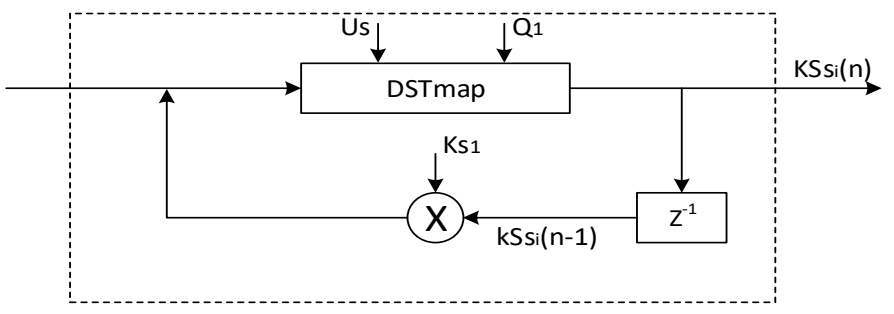

Fig. 8: The structure of the Chaotic System

3.3 Keyed hash functions based on two-layer $C N N$ structure (Structure 1)

The general architecture of the proposed keyed hash function is composed of the defined Chaotic System and two-layer $C N N$ (Fig. 9) [37]. Each layer is composed of 8 neurons, where each one uses a chaotic activation function (Figures 10 and 11). The chaotic activation function consists of two xored chaotic maps: a Discrete Skew Tent map (DSTmap) and a Discrete Piecewise Linear Chaotic map (DPWLCmap) $[43,44,67]$. Each map is iterated T times (by experiment, we choose the transient phase $\operatorname{tr}=30$ for Structure 1 and $\operatorname{tr}=20$ for Structure 2), before generating the first useful sample for maintaining the randomness of the output. The outputs of the DPWLCmap are defined as follows:

$$
\begin{aligned}
& K S p(n)=D P W L C \operatorname{map}(K S p(n-1), Q 2) \\
& = \begin{cases}2^{N} \times \frac{K S p(n-1)}{Q 2} & \text { if } 0<K S p(n-1) \leq Q 2 \\
2^{N} \times \frac{K S p(n-1)-Q 2}{2^{N-1}-Q 2} & \text { if } Q 2<K S p(n-1) \leq 2^{N-1} \\
2^{N} \times \frac{2^{N}-K S p(n-1)-Q 2}{2^{N-1}-Q 2} & \text { if } 2^{N-1}<K S p(n-1) \leq 2^{N}-Q 2 \\
2^{N} \times \frac{2^{N}-K S p(n-1)}{Q 2} & \text { if } 2^{N}-Q 2<K S p(n-1) \leq 2^{N}-1 \\
2^{N}-1-Q 2 & \text { otherwise }\end{cases}
\end{aligned}
$$

where $Q 2$ is the control parameter of DPWLCmap and ranges from 1 to $2^{N-1}$ $(\mathrm{N}=32$ bits).

It should be noted that in the proposed structures, the padded message $M$ is divided into $q$ blocks, where $M_{i}(1 \leq i \leq q)$ is the $i^{\text {eme }}$ input block of the message $M, K M_{i}(0 \leq i \leq q-1)$ is the $i^{\text {eme }}$ key, and $H M_{i}(1 \leq i \leq q)$ is the $i^{\text {eme }}$ hash value of block $M_{i}(1 \leq i \leq q)$. For the first block $M_{1}, K=K M_{0}$ is the secret key [44]. For the final block $M_{q}, h$ is the final hash value of the entire message $M$ (Fig. 12).

Detailed description of the two-layer CNN hash function: The detailed structure of the $i^{\text {eme }}$ block in the proposed two-layer $C N N$ hash function using MiyaguchiPreneel output scheme, as an example, is given in Fig. 10. Each of the input and output layers has 8 neurons. For each block $M_{i}$ at the input layer, each neuron has 8 input-data: $P_{j}(j=0, \ldots, 7)$ for neuron $0, P_{j}(j=8, \ldots, 15)$ for neuron 1 and so on 


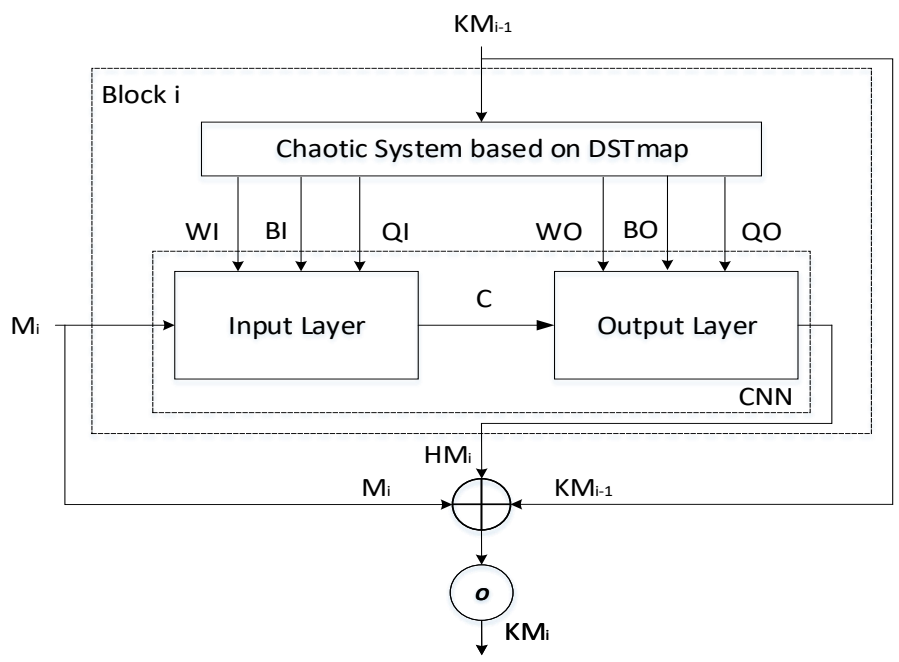

Fig. 9: The structure of the $i^{\text {eme }}$ block in the proposed keyed hash function based on two-layer $C N N$ with $M P$ output scheme

until reaching $P_{j}(j=56, \ldots, 63)$ for neuron 7 . Each $P_{j}(j=0, \ldots, 63)$ is weighted by $W I_{j}(j=0, \ldots, 63)$, where both are the samples (integer values) of 32 bits length. The Chaotic System generates the necessary samples (Key Stream $(K S)$ ) to supply the $C N N$ of each block $i$, which is as follows:

$$
K S=\{W I, B I, Q I, W O, B O, Q O\}
$$

and its size is written as:

$$
\begin{aligned}
|K S| & =|W I|+|B I|+|Q I|+|W O|+|B O|+|Q O| \\
& =176 \text { samples }
\end{aligned}
$$

where $|W I|=64$ samples, $|B I|=8$ samples, $|Q I|=16$ samples, $|W O|=64$ samples, $|B O|=8$ samples, and $|Q O|=16$ samples, each of the 32 bits length.

The chaotic activation function of each neuron $k(k=0, \ldots, 7)$ for the input layer is now explained as an example, (the activation function for the output layer has similar description). As we can see in Fig. 11, the first four inputs $P_{j}(j=8 k, \ldots, 8 k+3)$ are weighted by the $W I_{j}(j=8 k, \ldots, 8 k+3)$ and then added together with the bias $B I_{k}$ (weighted by 1) to form the input of DSTmap. The second four inputs $P_{j}(j=8 k+4, \ldots, 8 k+7)$ are weighted by $W I_{j}(j=8 k+4, \ldots, 8 k+7)$ and then added together with the same bias $B I_{k}$ to form the input of DPWLCmap. $Q I_{k, 1}$ and $Q I_{k, 2}$ are the control parameters of DSTmap and DPWLCmap, respectively. The biases $B I_{k}$ are necessary in case the input message is null.

The outputs of the chaotic activation function are denoted $C_{k}$ for the input layer, which is given by equation 11 , and $H_{k}$ for the output layer, which is given by 


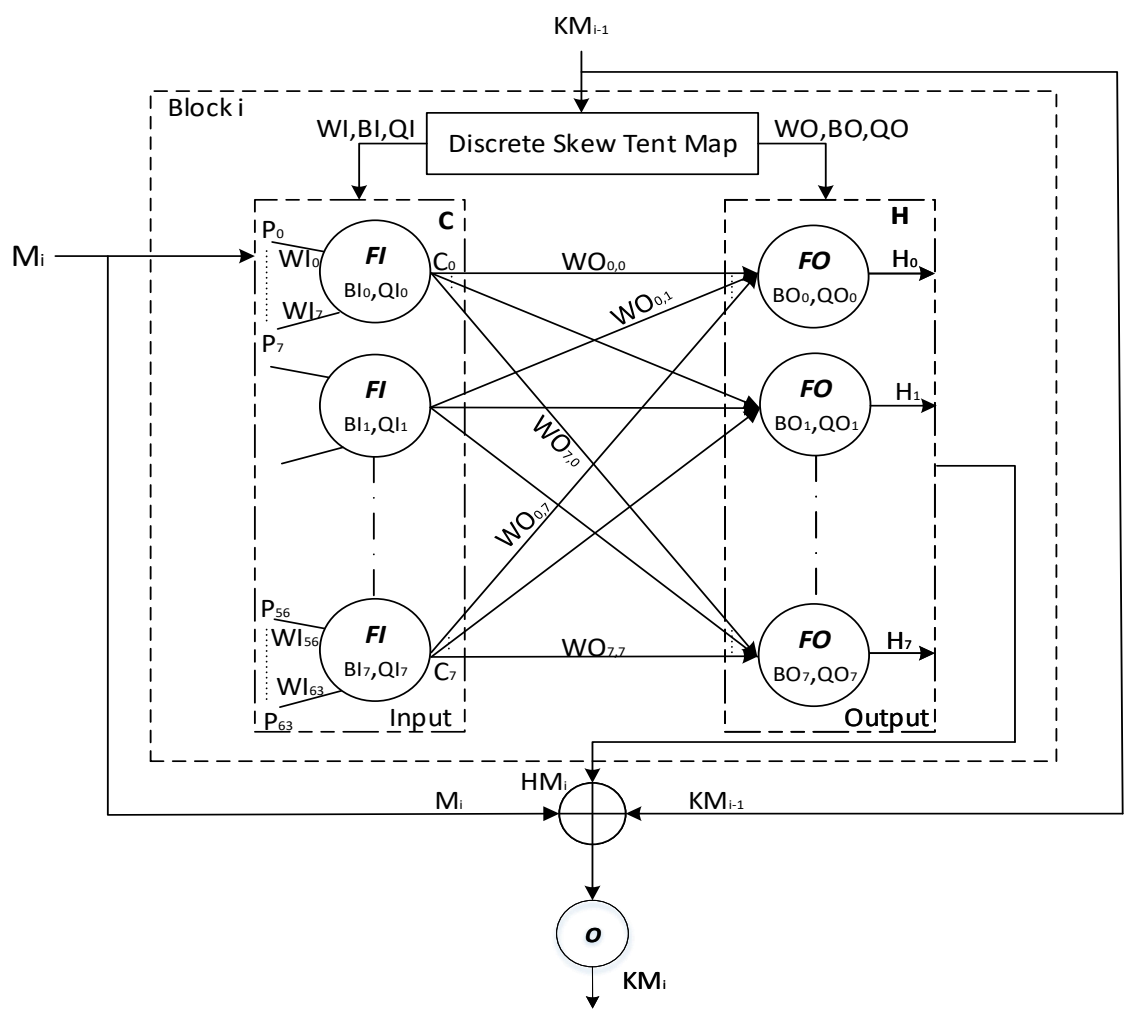

Fig. 10: A detailed structure of the $i^{\text {eme }}$ block in the proposed keyed hash function based on two-layer $C N N$ with $M P$ output scheme

equation 12 .

$$
\begin{aligned}
& C_{k}=\bmod \left\{[F 1+F 2], 2^{N}\right\} \text { where } \\
& \left\{\begin{array}{r}
F 1=D S T \operatorname{map}\left\{\bmod \left(\left[\sum_{j=8 k}^{8 k+3}\left(W I_{j} \times P_{j}\right)\right]+B I_{k}, 2^{N}\right),\right. \\
\left.Q I_{k, 1}\right\} \\
F 2=D P W L C \operatorname{map}\left\{\operatorname { m o d } \left(\left[\sum_{j=8 k+4}^{8 k+7}\left(W I_{j} \times P_{j}\right)\right]+B I_{k},\right.\right. \\
\left.\left.2^{N}\right), Q I_{k, 2}\right\}
\end{array}\right.
\end{aligned}
$$




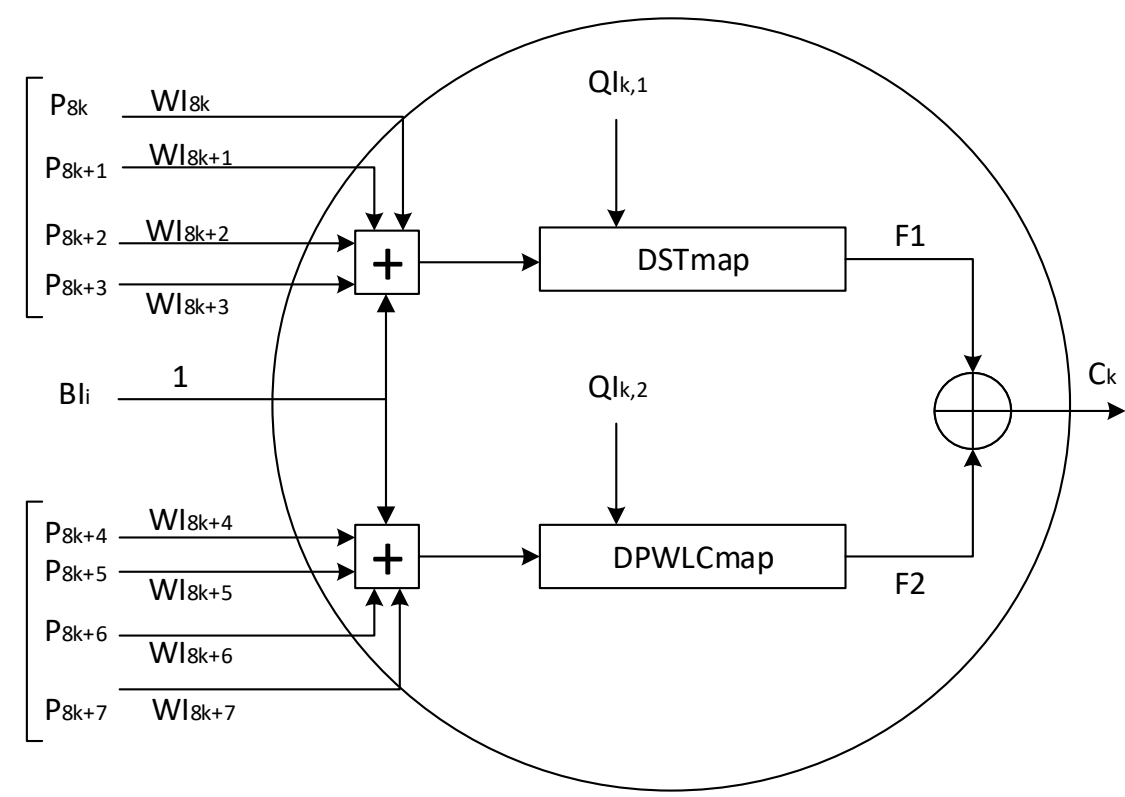

Fig. 11: A detailed structure of the $k^{e m e}$ neuron in input layer of the two proposed hash functions

$$
\begin{aligned}
& H_{k}=\bmod \left\{\left[G 1+G 2,2^{N}\right]\right\} \text { where } \\
& \left\{\begin{array}{r}
G 1=D S T \operatorname{map}\left\{\bmod \left(\left[\sum_{j=0}^{3}\left(W O_{k, j} \times C_{j}\right)\right]+B O_{k}, 2^{N}\right),\right. \\
\left.Q O_{k, 1}\right\} \\
G 2=D P W L C \operatorname{map}\left\{\operatorname { m o d } \left(\left[\sum_{j=4}^{7}\left(W O_{k, j} \times C_{j}\right)\right]+B O_{k},\right.\right. \\
\left.\left.2^{N}\right), Q O_{k, 2}\right\}
\end{array}\right.
\end{aligned}
$$

where $k=0,1, \ldots, 7$.

The outputs $C_{k}$ of the input layer, weighted by $W O_{k, k}(k=0, \ldots, 7)$, and the output biases $B O_{k}(k=0, \ldots, 7)$, weighted by 1 , are the inputs of the activation function of the output layer. Both $W O_{k, k}$ and $B O_{k}$ are samples of 32 bits length. For each neuron, DSTmap and DPWLCmap are iterated once. The output $H M_{i}(i=1, \ldots, q)$ of each block is the concatenation vector of $H_{k}(k=0, \ldots, 7)$ (Fig. 12). Then, the final hash value of length 256 bits is given by the following equation:

$$
\begin{aligned}
h & =O\left[K M_{q-1} \oplus H M_{q} \oplus M_{q}\right] \\
& =O\left[\left(K M_{q-2} \oplus H M_{q-1} \oplus M_{q-1}\right) \oplus H M_{q} \oplus M_{q}\right] \\
& =\ldots \\
& =O\left[\left(K \oplus H M_{1} \oplus M_{1}\right) \oplus H M_{2} \oplus M_{2} \oplus \ldots \oplus H M_{q} \oplus M_{q}\right]
\end{aligned}
$$

where $O$ is the Least Significant Bit $(L S B)$ output function. 


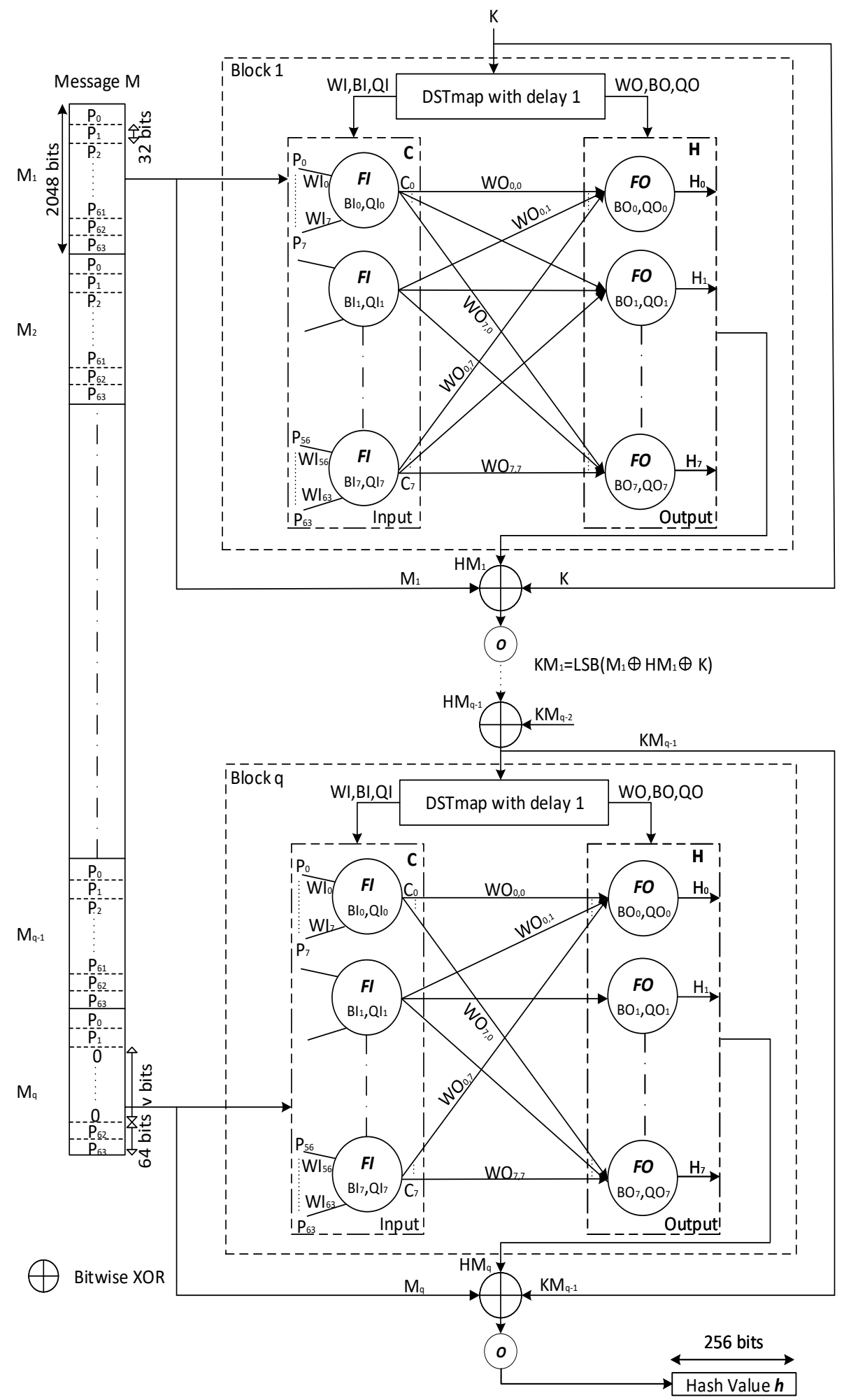

Fig. 12: The proposed keyed hash function based on two-layer $C N N$ with $M P$ output scheme 
3.4 Keyed hash functions based on one-layer $C N N$ with Non-Linear output layer (Structure 2)

Thus, to efficiently increase the hash throughput while keeping the necessary security requirements, we replace the output layer neural network of Fig. 10 by a combination of non-linear functions used in the standard SHA-2. However in our implementation, the round constant $K_{i}(i=0, \ldots, 63)$ and the message schedule array $W_{i}(i=0, \ldots, 63)$ are not useful (Fig. 13). As we can see in the figure 13 , the non-linear functions take 832 -bit inputs $D_{k}(k=0, \ldots, 7)$ and generates 832 -bit outputs $H_{k}(k=0, \ldots, 7)$. The four boxes $(C h, M a, \Sigma 0$, and $\Sigma 1)$ combine the input data in non-linear ways to generate $H_{0}$ and $H_{4}$, while the other outputs $H_{k}(k=1,2,3,5,6,7)$ are connected directly to $D_{k}$, which is as follows: $H_{k}=D_{k-1}(k=1,2,3,5,6,7)$. These non-liner functions are defined as follow [3]:

$$
\left\{\begin{array}{l}
C h\left(D_{4}, D_{5}, D_{6}\right)=\left(D_{4} \wedge D_{5}\right) \oplus\left(\neg D_{4} \wedge D_{6}\right) \\
\operatorname{Ma}\left(D_{0}, D_{1}, D_{2}\right)=\left(D_{0} \wedge D_{1}\right) \oplus\left(D_{0} \wedge D_{2}\right) \oplus\left(D_{1} \wedge D_{2}\right) \\
\Sigma 0\left(D_{0}\right)=\operatorname{ROTR}^{2}\left(D_{0}\right) \oplus \operatorname{ROTR}^{13}\left(D_{0}\right) \oplus \operatorname{ROTR}^{22}\left(D_{0}\right) \\
\Sigma 1\left(D_{4}\right)=\operatorname{ROTR}^{6}\left(D_{4}\right) \oplus \operatorname{ROTR}^{11}\left(D_{4}\right) \oplus \operatorname{ROTR}^{25}\left(D_{4}\right) \\
\operatorname{ROTR}^{n}(x)=(x \gg n) \vee(x \ll(32-n))
\end{array}\right.
$$

where $\wedge:$ : AND logic, $\neg:$ NOT logic, $\oplus:$ XOR logic, $\vee:$ OR logic, $\gg$ : Binary Shift Right operation, and $\ll$ : Binary Shift Left operation.

Detailed description of One-Layer CNN followed by NL functions: The structure of the proposed $C N N$ is given in Fig. 14. To supply the $C N N$, the Chaotic System generates the necessary samples (Key Stream $(K S)$ ) of each block $i$, which are as follows:

$$
K S=\{W I, B I, Q I, W O\}
$$

and its size is given as follows:

$$
\begin{aligned}
|K S| & =|W I|+|B I|+|Q I|+|W O| \\
& =96 \text { samples }
\end{aligned}
$$

where $|W I|=64$ samples, $|B I|=8$ samples, $|Q I|=16$ samples, and $|W O|=$ 8 samples, each of 32 bits length. The outputs $C_{k}(k=0, \ldots, 7)$ of the chaotic activation function given by equation 11 are weighted by $W O_{k, k}(k=0, \ldots, 7)$ to form the inputs of the $N L$ layer. The outputs $H_{k}(k=0, \ldots, 7)$ are given by equation 17.

$$
\left\{\begin{aligned}
H_{0}= & C h\left(D_{4}, D_{5}, D_{6}\right) \oplus D_{7} \oplus \Sigma 1\left(D_{4}\right) \oplus M a\left(D_{0}, D_{1}, D_{2}\right) \\
& \oplus \Sigma 0\left(D_{0}\right) \\
H_{1}= & D_{0}, H_{2}=D_{1}, H_{3}=D_{2} \\
H_{4}= & C h\left(D_{4}, D_{5}, D_{6}\right) \oplus D_{7} \oplus \Sigma 1\left(D_{4}\right) \oplus D_{3} \\
H_{5}= & D_{4}, H_{6}=D_{5}, H_{7}=D_{6}
\end{aligned}\right.
$$

We iterate the non-linear functions until the necessary security requirements are met. From experimental results (given in performance analysis paragraph), the number of rounds $r$ equals to 8 , which is sufficient. The final hash value $h$ of length 256 bits is given in equation 13 . 


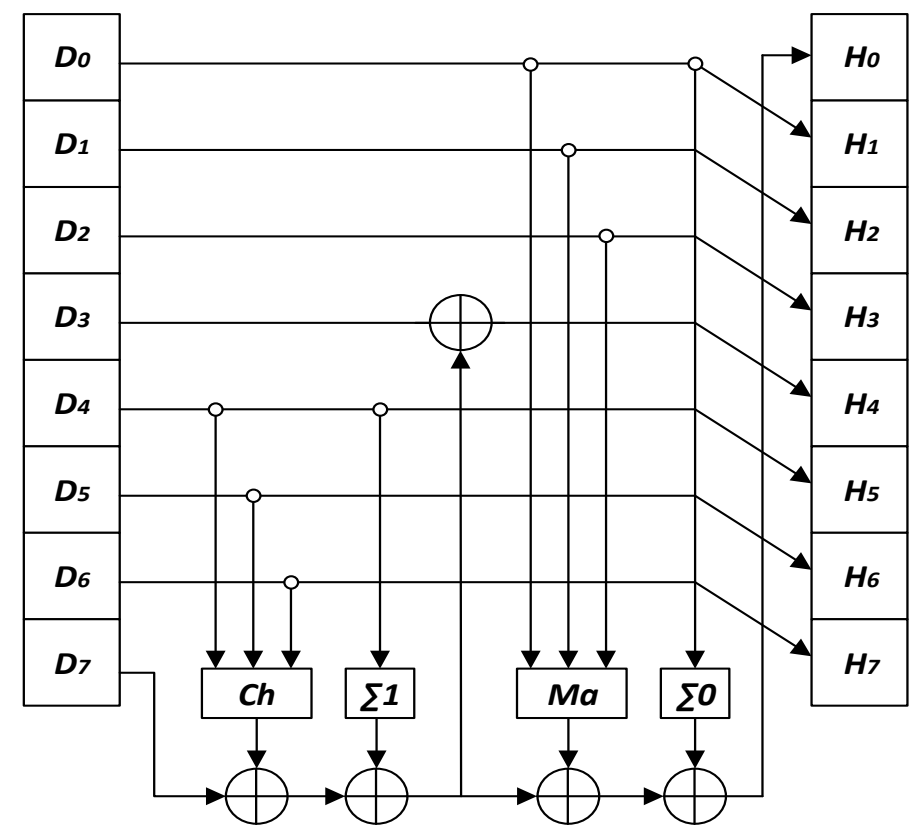

Bitwise XOR

Fig. 13: Non-linear functions

\section{Performance analysis}

To evaluate the performance, in terms of cryptanalysis and hash throughput, of the two proposed structures for each suggested output schemes, we perform the following experiments and analysis. Then, we compare their performance with most chaos-based hash functions in the literature and SHA-2. First, the one-way property (preimage resistance) is showed and then the statistical tests, the brute force, and cryptanalytical attacks of the proposed hash functions are analyzed (Fig. 15).

\subsection{One-way property:}

In the two proposed structures, we will show that it is extremely difficult to compute the message $M$ and the secret key $K$ when only the hash value $h$ is known. For the first structure, the hash $H$ is written in a general form, which is as follows (equations 11 and 12):

$$
\begin{aligned}
H & =G[(W O \times C+B O), Q O] \\
& =G[(W O \times F((W I \times P+B I), Q I), Q O)]
\end{aligned}
$$




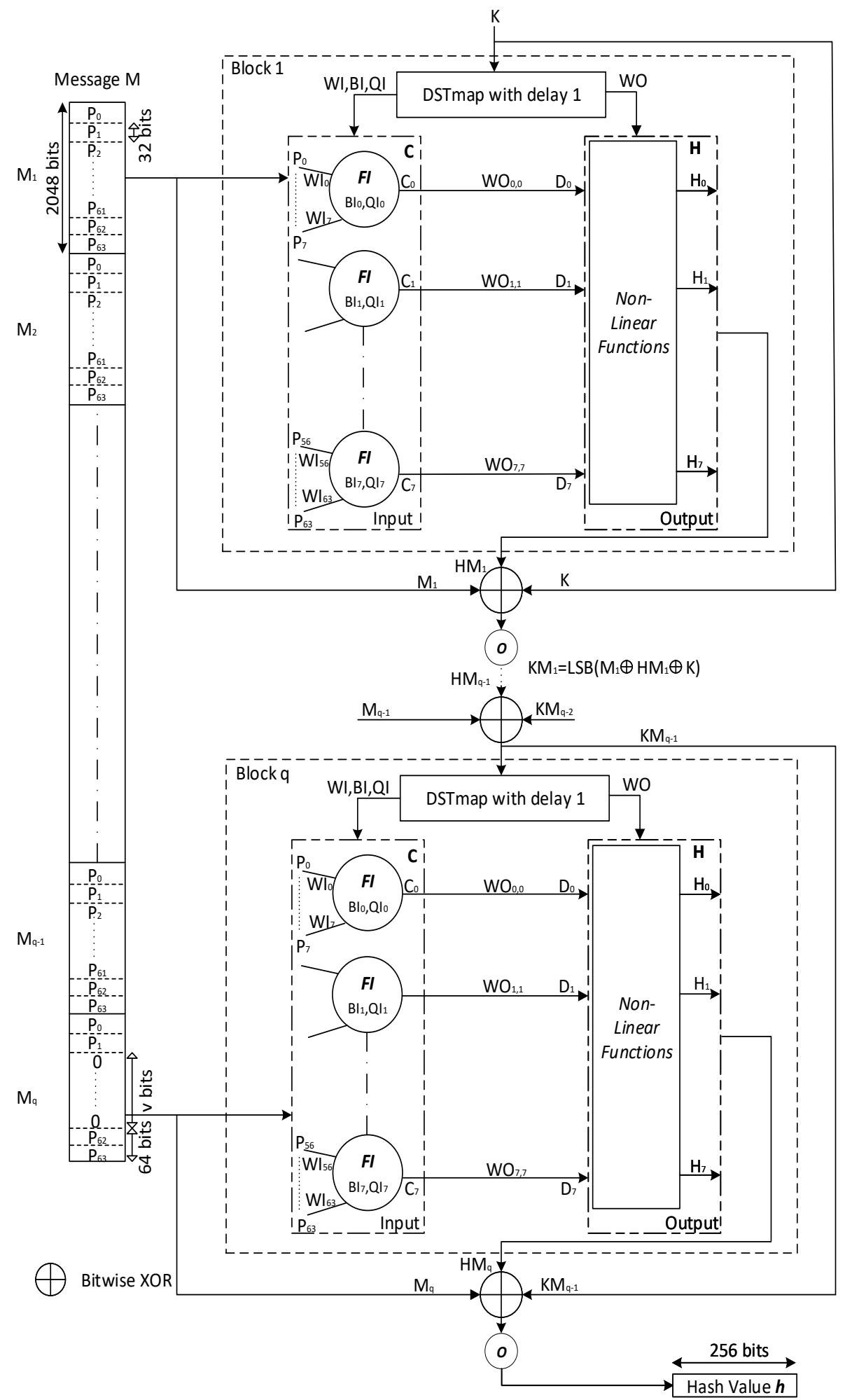

Fig. 14: The proposed keyed hash function based on one-layer $N L C N N$ with $M P$ output scheme 


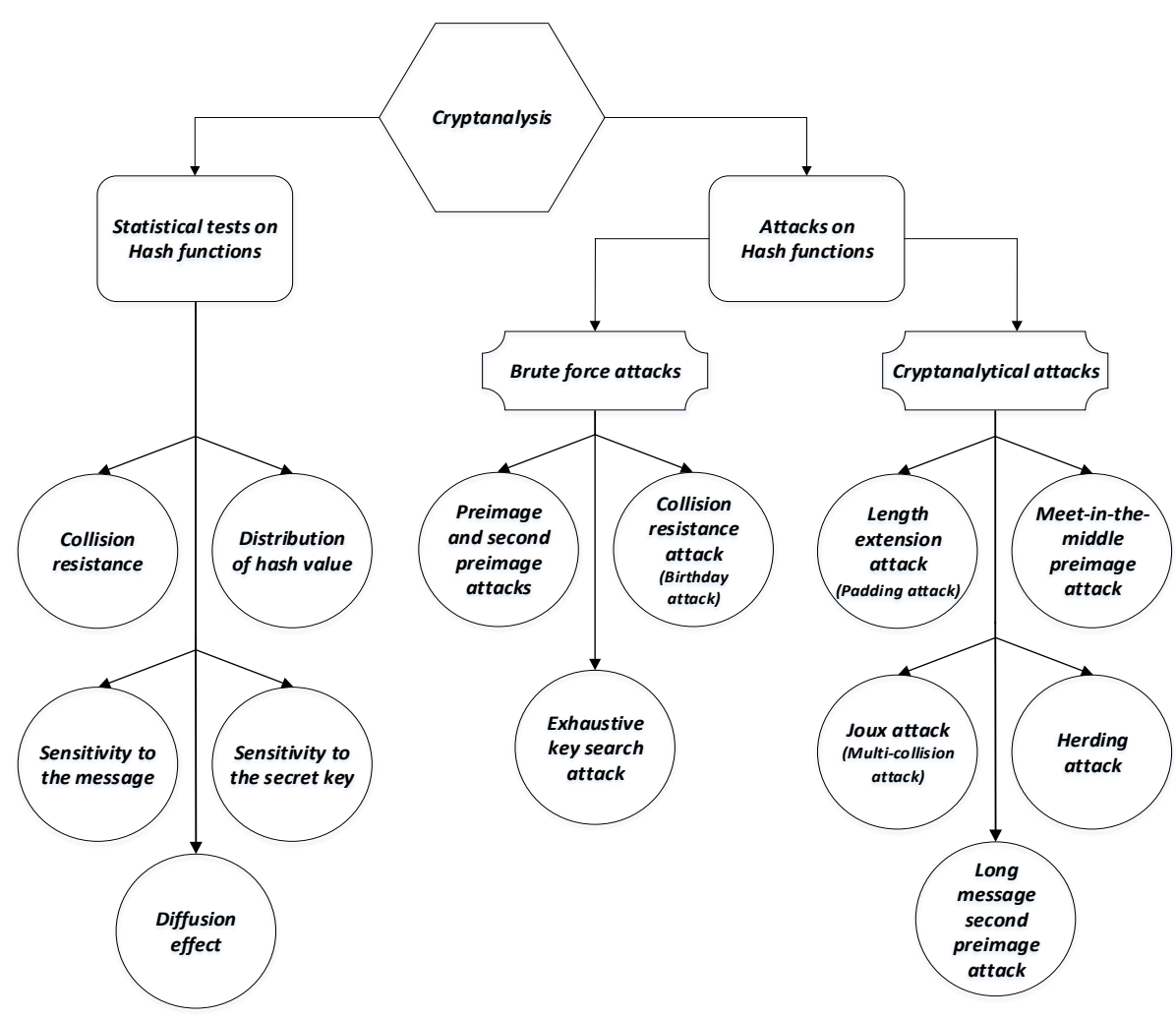

Fig. 15: Cryptanalysis: Statistical tests and attacks on hash functions

For the second structure, the hash $H$ can be written as follows:

$$
\begin{aligned}
H & =N L^{r}(W O \times C) \\
& =N L^{r}[W O \times F((W I \times P+B I), Q I)]
\end{aligned}
$$

A brute force attack, as defined in sub-section 4.3.1, tries for a given secret key $K$ to find a message $M$, of which its hash is equal to a given hash value. The attacker needs to try, on average, $2^{u-1}$ values of $M$, to find the desired hash value $h$. As $u$ is the length of the hash value equal to 256 bits in the two proposed structures, then according to today's computing ability, this attack is infeasible [7, 8, 21, 22].

\subsection{Statistical tests}

This paragraph lists down the analysis of the following tests: Collision resistance, Distribution of hash value, Sensitivity of hash value $h$ to the message $M$, Sensitivity of hash value $h$ to the secret key $K$, and Diffusion effect. 


\subsubsection{Analysis of collision resistance}

This test is usually conducted to evaluate the quantitative analysis of collision resistance [5,7]. First, the hash value $h$ of a random message is generated and stored in the ASCII format. Next, a bit in the message is randomly selected, toggled, and then a new hash value $h^{\prime}$ is generated and stored in the ASCII format. The two hash values are represented by: $h=\left\{c_{1}, c_{2}, \ldots, c_{s}\right\}$ and $h^{\prime}=\left\{c_{1}^{\prime}, c_{2}^{\prime}, \ldots, c_{s}^{\prime}\right\}$, where $c_{i}$ and $c_{i}^{\prime}$ are the $i^{t h}$ ASCII character of the two hash values $h$ and $h^{\prime}$, respectively. The size $s$ of the hash value in the ASCII code is equal to $s=\frac{u}{k=8}=32$ characters. The two hash values are compared with each other and the number of characters with the same value at the same location, namely the number of hits $\omega$, is counted according to the following:

$$
\begin{aligned}
& \omega=\sum_{i=1}^{s=32} f\left(T\left(c_{i}\right), T\left(c_{i}^{\prime}\right)\right) \\
& \text { where } f(x, y)=\left\{\begin{array}{ll}
1 & \text { if } x=y \\
0 & \text { if } x \neq y
\end{array}\right\}
\end{aligned}
$$

where the function $T($.$) converts the entries to their equivalent decimal values.$ For $\mathrm{J}$ independent experiments and under the assumption of uniform and random distribution of hash value, the theoretical number of tests denoted by $W_{J}(\omega)$ with a number of hits $\omega=0,1,2, \ldots, s$, is given by [19]:

$$
W_{J}(\omega)=J \times \operatorname{Prob}\{\omega\}=J \frac{s !}{\omega !(s-\omega) !}\left(\frac{1}{2^{k}}\right)^{\omega}\left(1-\frac{1}{2^{k}}\right)^{s-\omega}
$$

Thus, to find the optimal number of round $r$ for Structure 2, we calculate, using the equation 20 , the number of hits $\omega$ according to $r(r=1,2,4,8,16,24)$ in the worst case, where the number of tests $\mathrm{J}=2048$ tests.

As we can see from the results obtained in Table 2, with $M M O$ output scheme, as an example, for $r=8$ rounds, there are zero hits for 1825 tests, one hit for 207 tests, two hits for 15 tests, and three hits for 1 test. For $r=24$ rounds, there are zero hits for 1817 tests, one hit for 225 tests, and two hits for 6 tests. Similar results are obtained for other output schemes as well. The number of rounds $r$ equals 8 , whereas 24 seems to be adequate for the three output schemes. We choose $r=24$, for more robustness and the number $r=8$ is a compromise between robustness and hash throughput.

Table 3 represents the number of obtained hits $\omega$, for the proposed structures for the three output schemes, with $\mathrm{J}=2048$ tests and for $r=8,24$ rounds for Structure 2. We remark that, for $r=8$ rounds, the obtained results with Structure $\mathbf{2}$ are similar to the results obtained with Structure 1, irrespective of the considered output scheme. For $r=24$ rounds, the obtained results with Structure 2, as are slightly bit better than that of Structure $\mathbf{1}$.

Thus, to evaluate the influence of the test number $\mathrm{J}(\mathrm{J}=512,1024$, and 2048 tests) on the number of hits, we calculate $\omega$ for the proposed structures with $M P$ output scheme, and for $r=8,24$ rounds for the second structure. The obtained results presented in Table 4 for Structures 1 and $\mathbf{2}$ with $r=8$ rounds are similar, while with $r=24$ rounds of Structure 2, the number of hits is smaller than that of the other cases. We remark that the number of hits increases with the number 


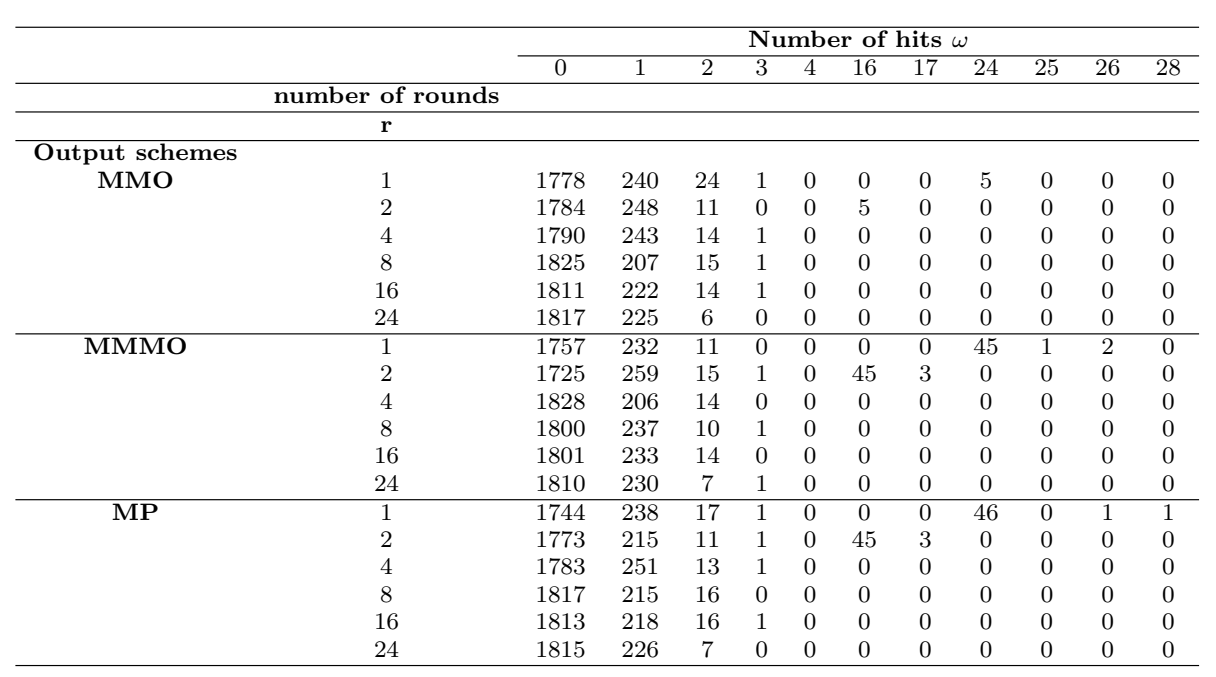

Table 2: Number of hits $\omega$ according to the number of rounds $r$ of Structure 2 for 2048 tests

of tests J. These results are in sync with the theoretical values of $W_{J}(\omega)$ calculated from equation 21 and are represented in Table 5 .

The collision resistance is also quantified by the absolute difference $d$ of two hash values given by equation 22 . We evaluated and presented the mean, mean/character, minimum, and maximum of $d$ for the two proposed hash functions in Tables 6 and 7 .

$$
d=\sum_{i=1}^{s=32}\left|T\left(c_{i}\right)-T\left(c_{i}^{\prime}\right)\right|
$$

From the results given in Table 6 for $\mathrm{J}=2048$ tests, we observe that the mean/character value with the $M M O$ output scheme for Structure $1($ mean/character $=85.04)$ and Structure $2-r=24$ rounds (mean/character $=85.81$ ) are close to the expected value $85 . \overline{3}$ given in equation 23 . The results presented in Table 7 with $\mathrm{J}(\mathrm{J}$ $=512,1024$, and 2048 tests) show that, when $\mathrm{J}$ is increasing, the mean/character converge to the expected value $E$. For two hash, i.e., $h=\left\{c_{1}, c_{2}, \ldots, c_{s}\right\}$ and $h^{\prime}=\left\{c_{1}^{\prime}, c_{2}^{\prime}, \ldots, c_{s}^{\prime}\right\}$, with independent and uniformly distributed ASCII character having equal probabilities, the expected value of the mean/character is calculated by [68]:

$$
E\left[T\left(c_{i}\right)-T\left(c_{i}^{\prime}\right)\right]=\frac{1}{3} \times L=85 . \overline{3}
$$

where $T\left(c_{i}\right)$ and $T\left(c_{i}^{\prime}\right) \in\{0,1,2, \ldots, 255\}$ and $L=256$ ( $\mathrm{L}$ is the number of levels).

\subsubsection{Distribution of hash value}

A hash function $H$ should produce uniform distribution of hash value $h$. To verify this property, we perform the following test: for a given message $M$, With the 


\begin{tabular}{cccccc}
\hline & Output schemes & \multicolumn{4}{c}{ Number of hits $\omega$} \\
\cline { 2 - 6 } & & 0 & 1 & 2 & 3 \\
\hline Structure 1 & $M M O$ & 1833 & 200 & 15 & 0 \\
& $M M M O$ & 1799 & 237 & 12 & 0 \\
& $M P$ & 1803 & 232 & 13 & 0 \\
\hline \hline Structure 2 & $M M O$ & 1825 & 207 & 15 & 1 \\
r = 8 & $M M M O$ & 1800 & 237 & 10 & 1 \\
& $M P$ & 1817 & 215 & 16 & 0 \\
\hline Structure 2 & $M M O$ & 1817 & 225 & 6 & 0 \\
r = 24 & $M M M O$ & 1810 & 230 & 7 & 1 \\
& $M P$ & 1815 & 226 & 7 & 0 \\
\hline
\end{tabular}

Table 3: Number of hits $\omega$ regarding the proposed structures with the three output schemes for 2048 tests

\begin{tabular}{cccccc}
\hline & Number of tests & \multicolumn{4}{c}{ Number of hits $\omega$} \\
\cline { 2 - 6 } & & 0 & 1 & 2 & 3 \\
\hline Structure 1 & 512 & 444 & 64 & 4 & 0 \\
& 1024 & 905 & 111 & 8 & 0 \\
& 2048 & 1803 & 232 & 13 & 0 \\
\hline \hline Structure 2 & 512 & 446 & 62 & 4 & 0 \\
$\quad \mathbf{r}=\mathbf{8}$ & 1024 & 899 & 117 & 8 & 0 \\
& 2048 & 1817 & 215 & 16 & 0 \\
\hline Structure 2 & 512 & 452 & 58 & 2 & 0 \\
r = 24 & 1024 & 905 & 116 & 3 & 0 \\
& 2048 & 1815 & 226 & 7 & 0 \\
\hline
\end{tabular}

Table 4: Number of hits $\omega$ of the proposed structures with $M P$ output scheme for $\mathrm{J}=512,1024$, and 2048 tests

\begin{tabular}{ccccccc}
\hline & & \multicolumn{5}{c}{$\omega$} \\
\cline { 3 - 7 } & & 0 & 1 & 2 & 3 & 32 \\
\hline $\mathrm{J}$ & 512 & 451.72 & 56.68 & 3.44 & 0.13 & $4.42 \times 10^{-75}$ \\
& 1024 & 903.45 & 113.37 & 6.89 & 0.27 & $8.84 \times 10^{-75}$ \\
& 2048 & 1806.91 & 226.74 & 13.78 & 0.54 & $1.76 \times 10^{-74}$ \\
\hline
\end{tabular}

Table 5: Theoretical values of the number of hits $\omega$ according to the number of tests $\mathrm{J}$

\begin{tabular}{cccccc}
\hline & Output schemes & Mean & Mean/character & Minimum & Maximum \\
\hline Structure 1 & $M M O$ & 2721.43 & 85.04 & 1736 & 3723 \\
& $M M M O$ & 2764.05 & 86.37 & 1829 & 3757 \\
& $M P$ & 2633.17 & 82.28 & 1471 & 3779 \\
\hline \hline Structure 2 & $M M O$ & 2616.94 & 81.77 & 1559 & 3574 \\
r= 8 & $M M M O$ & 2854.76 & 89.21 & 1845 & 4195 \\
& $M P$ & 2861.93 & 89.43 & 1707 & 3951 \\
\hline Structure 2 & $M M O$ & 2746.07 & 85.81 & 1696 & 3807 \\
r= 24 & $M M M O$ & 2856.03 & 89.25 & 1545 & 3981 \\
& $M P$ & 2615.44 & 81.73 & 1540 & 3671 \\
\hline
\end{tabular}

Table 6: Mean, Mean/character, Minimum, and Maximum of the absolute difference $d$ for the proposed structures with the three output schemes and $J=2048$ tests 


\begin{tabular}{cccccc}
\hline & Number of tests & Mean & Mean/character & Minimum & Maximum \\
\hline Structure 1 & 512 & 2637.00 & 82.40 & 1471 & 3779 \\
& 1024 & 2637.99 & 82.43 & 1471 & 3779 \\
\hline \hline Structure 2 & 2048 & 2633.17 & 82.28 & 1471 & 3779 \\
r= 8 & 512 & 2872.23 & 89.75 & 1828 & 3872 \\
& 1024 & 2868.04 & 89.62 & 1707 & 3951 \\
\hline Structure 2 & 2048 & 2861.93 & 89.43 & 1707 & 3951 \\
r= 24 & 512 & 2603.32 & 81.35 & 1764 & 3671 \\
& 1024 & 2620.85 & 81.90 & 1626 & 3671 \\
& 2048 & 2615.44 & 81.73 & 1540 & 3671 \\
\hline
\end{tabular}

Table 7: Mean, Mean/character, Minimum, and Maximum of the absolute difference $d$ for the proposed structures with $M P$ output scheme and $\mathrm{J}=512,1024$, and 2048 tests

wide application of Internet and computer technique, information security becomes more and more important. As we know, hash function is one of the cores of cryptography and plays an important role in information security. Hash function takes a message as input and produces an output referred to as a hash value. A hash value serves as a compact representative image (sometimes called digital fingerprint) of input string and can be used for data integrity in conjunction with digital signature schemes., we calculate its hash value $h$, for the proposed Structure 1 with $M P$ output scheme, before drawing two-dimensional graphs. The first graph shows the ASCII values of the message according to their index positions (Fig. 16a). The second graph exhibits the hexadecimal values of the hash value $h$ according to their index positions (Fig. 16b). As we can see, the distribution of original message is mostly localized around a small area, while the distribution of hexadecimal values spreads around the entire area. This property of hash value $h$ must be true under the worst case of null input message (Figures 16c and 16d). Similar results are obtained for the two proposed hash functions with their different output schemes.

\subsubsection{Sensitivity of hash value $h$ to the message $M$}

An efficient hash function $H$ should be extremely sensitive to any input message $M$, which means that any slight change in the input message should produce a completely different hash value $h_{i}$. To verify this property, we calculate, for a given secret key $K$, the hash value $h_{i}$ in hexadecimal format, the number of bits changed $B_{i}\left(h, h_{i}\right)$ (bits), and the sensitivity of the hash value $h$ to the original message $M$ measured by Hamming Distance $H D_{i}\left(h, h_{i}\right)(\%)$ is given as follows:

$$
\begin{aligned}
& B_{i}\left(h, h_{i}\right)=\sum_{k=1}^{|h|}\left[h(k) \oplus h_{i}(k)\right] \text { bits } \\
& H D_{i}\left(h, h_{i}\right)_{\%}=\frac{B_{i}\left(h, h_{i}\right)}{|h|} \times 100 \%
\end{aligned}
$$

The message variants are obtained under the following conditions:

Condition 1: The original message $M$ is the one given in Sect. 4.2.2.

Condition 2: We change the first character $\mathbf{W}$ in the original message to $\mathbf{X}$. 


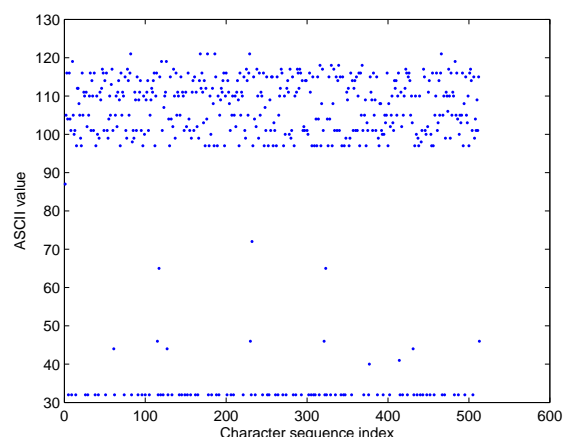

(a)

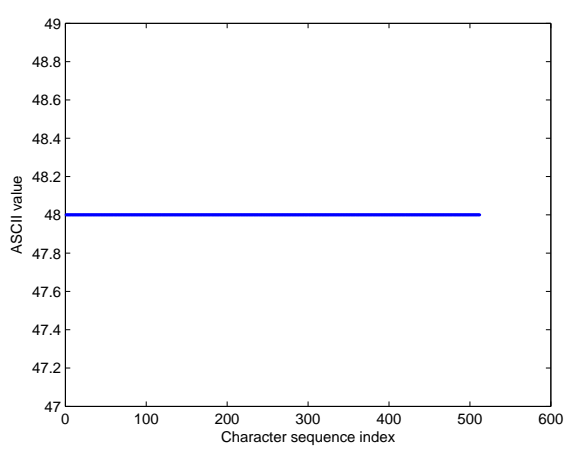

(c)

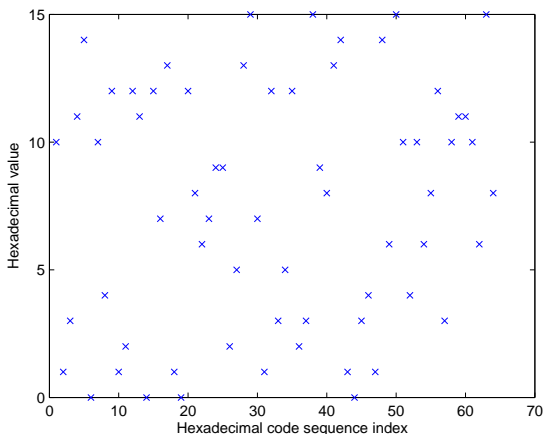

(b)

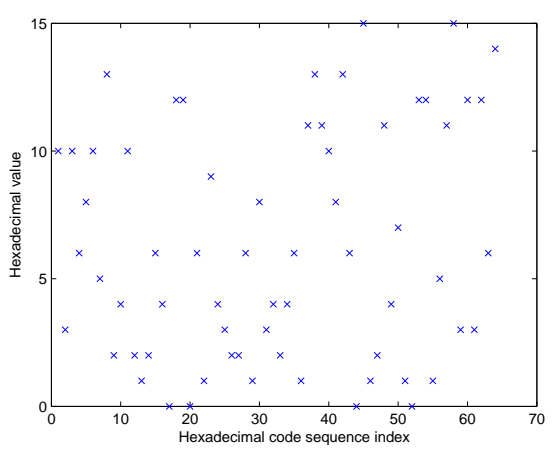

(d)

Fig. 16: Distribution of hash value for Structure 1 with $M P$ output scheme

Condition 3: We change the word With in the original message to Without. Condition 4: We change the dot at the end of the original message to comma. Condition 5: We add a blank space at the end of the original message.

Condition 6: We exchange the first message block $M_{1}$, With the wide application of Internet and computer technique, information security becomes more and more important. As we know, hash function is one of the cores of cryptography and plays an important role in information security. Hash function takes a mes, with the second message block $M_{2}$, sage as input and produces an output referred to as a hash value. A hash value serves as a compact representative image (sometimes called digital fingerprint) of input string and can be used for data integrity in conjunction with digital signature schemes.

In Tables 8, 9, and 10, we present the obtained results of $h_{i}, B_{i}$, and $H D_{i}(\%)$ under each condition for the two proposed hash functions with their output schemes, i.e., $M M O, M M M O$, and $M P$.

In Table 11, we reassessed the obtained results and even for a single test, the results were inside the normal range. Therefore, the proposed hash functions have 


\begin{tabular}{|c|c|c|c|c|}
\hline & Message variants & Hexadecimal hash values & $B_{i}$ & $H D_{i} \%$ \\
\hline \multirow[t]{7}{*}{ Structure 1} & 1 & bedf7967520105d114e2cdf3399f52394a53e276bb104307345bacf93e317ef6 & & \\
\hline & 2 & 48def8102016f2e3a5f8e7d8dc782b5b4e3e930cc207f925176ab87f380ad03d & 125 & 48.82 \\
\hline & 3 & d486760a20882b71746704d35ffdcd0f07c5ffe23cad86bd8117737205dd163c & 127 & 49.60 \\
\hline & 4 & b8bdc0f41686695f582a4d2e5b37f9b98813ab9c1cc42ba64024ee1769b422e 7 & 113 & 44.14 \\
\hline & 5 & e 82980358f548044d0328f613a640fe23d1cb8465325dc223a7881ae65ef360d & 136 & 53.12 \\
\hline & 6 & 76e33a9b2f6599542c557bdac7bee94f25dddbc615b222653201fd484ae8ce1c & 130 & 50.78 \\
\hline & Average & - & 126.2 & 49.29 \\
\hline \multirow{7}{*}{$\begin{array}{c}\text { Structure } 2 \\
r=8\end{array}$} & 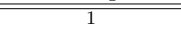 & 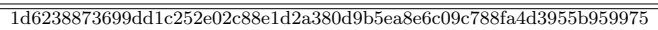 & & \\
\hline & 2 & 6ea75e2045e994639a7d547ece06a6a399397a6cc501f52ffe4d4727030bedb2 & 128 & 50 \\
\hline & 3 & 72dcdf42b4c6d47352b75a7f2a7bbb3d9144c519e99e10cdd1a04237433730bf & 131 & 51.17 \\
\hline & 4 & 308f5d0ce0c0a7b140cc7c179ae4697fba8ea270433c50b015095877c2047267 & 141 & 55.07 \\
\hline & 5 & ebeac17eb7b2d842ef21971f6c9da59771f7a0e0612ecd96e37a97691eb0c1cd & 135 & 52.73 \\
\hline & 6 & 1e56b053ebe94fc4eb36f8ed74981da9c01a861cdbe93b3c176ecfab8102a336 & 122 & 47.65 \\
\hline & Average & & 131.4 & 51.32 \\
\hline \multirow{7}{*}{$\begin{array}{c}\text { Structure } 2 \\
r=24\end{array}$} & 1 & af5e7ca7c83a72c77f0e9b7d47df11b0f66cadc86 & & \\
\hline & 2 & 46f051a065a716de24405e782adaccb29b3a85b0b75b34a9ba0757644bcdcc33 & 127 & 49.60 \\
\hline & 3 & 4f9c3863d40a2a1094d8d7483acc0724cbd9f2b68648db7fe 8c0609327c8f318 & 130 & 50.78 \\
\hline & 4 & $2 \mathrm{~b} 4 \mathrm{ff} 84285427 \mathrm{~b} 479 \mathrm{~d} 6948 \mathrm{~d} 20 \mathrm{dd} 00 \mathrm{eb} 389956 \mathrm{dd} 325894 \mathrm{~d} 6036 \mathrm{e} 510 \mathrm{~b} 99 \mathrm{~b} 20055 \mathrm{~d}$ & 126 & 49.21 \\
\hline & 5 & 15e6695fca52780d8694f83b0bba7b5fb43bc29329e78018287bd87776cdf459 & 132 & 51.56 \\
\hline & 6 & d5a2f663581034f865ba7a2bc93d29232b0f57f99f8d33a8ef50e1070c84ae88 & 133 & 51.95 \\
\hline & Average & & 129.6 & 50.62 \\
\hline
\end{tabular}

Table 8: Sensitivity of hash value to the message for the proposed structures with $M M O$ output scheme

\begin{tabular}{|c|c|c|c|c|}
\hline & Message variants & Hexadecimal hash values & $B_{i}$ & $H D_{i} \%$ \\
\hline \multirow[t]{7}{*}{ Structure 1} & 1 & 719adf0e0cdf5b149edc54efdbc09bb6df5a0ce3d3ac9bccc39ac5a64ea65531 & & \\
\hline & 2 & a9472c054759a $85 \mathrm{c} 0 \mathrm{c} 172 \mathrm{e} 27 \mathrm{bc} 1 \mathrm{~b} 957 \mathrm{f} 09488 \mathrm{c} 40329424 \mathrm{c} 48 \mathrm{aac} 1 \mathrm{~d} 1141 \mathrm{dd} 8297$ & 132 & 51.56 \\
\hline & 3 & 1bee2969559824929f8d53fda2c541288a4a04491a0a11670b3b907fa0d5dd91 & 119 & 46.48 \\
\hline & 4 & 27c29f1e040d922b31559e0e3f4e36edc9bdad55cf058d7f0eaa7a9f9eda6d98 & 124 & 48.43 \\
\hline & 5 & 65489772dff489621f3188237c1ff84c8bf686d7a4f5c6ff1e114b740c72c922 & 133 & 51.95 \\
\hline & 6 & 64755b1267f7243f2dbf243d698db2dd40ff63df7375f645886d064b2d05fdb2 & 135 & 52.73 \\
\hline & Average & & 128.6 & 50.23 \\
\hline \multirow{7}{*}{$\begin{array}{c}\text { Structure } 2 \\
\mathrm{r}=8\end{array}$} & 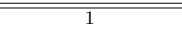 & 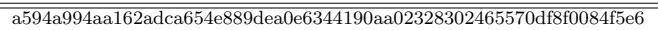 & & \\
\hline & 2 & 9d698ca7855b104a526a075a36cbf158da31c872257db0d8d589502f60a8115f & 135 & 52.73 \\
\hline & 3 & fe77f2939687110cc6f383ed0ac2990e89b513ed1425c2a2ded04ce8ab26331e & 129 & 50.39 \\
\hline & 4 & d906ae7eaf90974ce664e8adb535e71b798873bfdc77827e3715715bb6b5cbb5 & 113 & 44.14 \\
\hline & 5 & flea83b16b7fecd5d523573d35f52a 424e35a8dc38af6e013f9d2020f0825c35 & 136 & 53.12 \\
\hline & 6 & 29e7a1e00480ff09b86d357982d28ab641758c071ceela2095452cb583740194 & 121 & 47.26 \\
\hline & Average & & 126.8 & 49.53 \\
\hline \multirow{7}{*}{$\begin{array}{c}\text { Structure } 2 \\
\mathbf{r}=\mathbf{2 4}\end{array}$} & 1 & 6abbd825d6b17184a5fc558670f9f78d91b3812c899c8a062ef855507b4a81e5 & & \\
\hline & 2 & c7c8654da6fd4fb838f8f9bea4baa223b8298a1c1e0cda2181a23e612cbb8446 & 122 & 47.65 \\
\hline & 3 & Ofe4ee2f96a9092f539a4fd229466b381a794db148da178e635022d9a690eabf & 130 & 50.78 \\
\hline & 4 & 9b01f686addb2e2f6dbd7046b985b4ae1b5b39a7da3aec544ecb6c8efd310a00 & 128 & 50.00 \\
\hline & 5 & 9901ff0d69138df2f70a5930ede63447875c859830bc87e4164a83b083a6a193 & 131 & 51.17 \\
\hline & 6 & c5035924044140a2009837907fba710d05efbcbe12ff9c1d14d9090961bd054e & 113 & 44.14 \\
\hline & Average & . & 124.8 & 48.75 \\
\hline
\end{tabular}

Table 9: Sensitivity of hash value to the message for the proposed structures with $M M M O$ output scheme

high message sensitivity. These results were in sync with precision in the diffusion test, which was realized over a large number of tests.

\subsubsection{Sensitivity of hash value $h$ to the secret key $K$}

Thus, to evaluate the sensitivity of hash value $h$ to the secret key $K$, hash simulation experiments were conducted under five different conditions (the original input message $M$ is fixed), which are as follows:

Condition 1: The original secret key $K$ is used.

In each of these conditions, we flip the $L S B$ in the afore-mentioned initial conditions and parameters.

Condition 2: We change the initial condition $\boldsymbol{K} \boldsymbol{S} \boldsymbol{s}(\boldsymbol{O})$ in the secret key.

Condition 3: We change the parameter $\boldsymbol{K} \boldsymbol{s}$ in the secret key.

Condition 4: We change the initial condition $\boldsymbol{K S} \boldsymbol{S}(-\mathbf{1})$ in the secret key.

Condition 5: We change the control parameter $\boldsymbol{Q 1}$ in the secret key. 


\begin{tabular}{|c|c|c|c|c|}
\hline & Message variants & Hexadecimal hash values & $B_{i}$ & $H D_{i} \%$ \\
\hline \multirow[t]{7}{*}{ Structure 1} & 1 & a005e50f9673ecee6e80c07c550e53f8a950cb4a91176a2a340b5822ec2f28c4 & & \\
\hline & 2 & d4ecfadcc796f46d63762eb8f0c7af6233ded0d61ea901541db1f8890f999755 & 141 & 55.07 \\
\hline & 3 & 3f8b28e72a453ad31e798a60ec46b64ab4eb3e95674b28d535a5d2feb8a7cdd8 & 139 & 54.29 \\
\hline & 4 & b40f8be0ee3c28fc7c76578d6e8b49f56ea25aa0c2944475691746a7c2f23387 & 129 & 50.39 \\
\hline & 5 & c0f0b6c0fee17303c94ab30ad6d7b1ecd50d9606e4fab176e726b20a3c229b5d & 139 & 54.29 \\
\hline & 6 & 551eb7f04ec0ae2f0ceec2bb451a2b67682305697a0ffef418e221bdaad4a09c & 129 & 50.39 \\
\hline & Average & 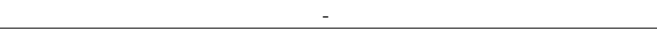 & 135.40 & 52.89 \\
\hline \multirow{7}{*}{$\begin{array}{c}\text { structure } 8 \\
r=8\end{array}$} & 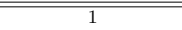 & 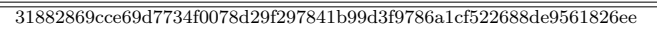 & & \\
\hline & 2 & d8da2ae1aacca231e26931237f8ba1388aef0faf2372dde8876d329564bb4f39 & 129 & 50.39 \\
\hline & 3 & 0b43925c8865869e7dde5c67cfd976f839bd8f5c8fda2814c2c61ce4c926b380 & 130 & 50.78 \\
\hline & 4 & d9e813e6f36a7a960664ab422b1eb1892be71f43a28229399bdcf51a5ab0df8d & 131 & 51.17 \\
\hline & 5 & d0f1dcbf0670f8a3ef2771d0f0d8404c6068ab43b303d1aa9e335d9a757ddb6b & 149 & 58.20 \\
\hline & 6 & $1441805 \mathrm{beb} 1753 \mathrm{~d} 9 \mathrm{c} 81 \mathrm{bd} 16 \mathrm{~d} 9059 \mathrm{f} 3 \mathrm{f} 2 \mathrm{e} 57752732 \mathrm{c} 1 \mathrm{f} 2 \mathrm{e} 539 \mathrm{ec} 606555 \mathrm{f} 2 \mathrm{~d} 9042$ & 137 & 53.51 \\
\hline & Average & & 135.2 & 52.81 \\
\hline \multirow{7}{*}{$\begin{array}{c}\text { Structure } 2 \\
\mathrm{r}=\mathbf{2 4}\end{array}$} & 1 & a86e4c2ff1450a08a173b2d9ef27d941fcb! & - & \\
\hline & 2 & 22e2025f1d0bdb5b20098e8f2d81a63b27e722c9e2eb521e87e00943f7af1dbe & 132 & 51.56 \\
\hline & 3 & 366d73069aa3e7238773a6ba39bbfc $29203 \mathrm{f} 28 \mathrm{ffd} 05 \mathrm{f} 8 \mathrm{fec} 06060 \mathrm{ececc} 54 \mathrm{fc} 2 \mathrm{e}$ & 113 & 44.14 \\
\hline & 4 & cd1fcb9c2c9alcaab20b4c8bf1ff18493533b42004d9f7741f957ab1850831db & 128 & 50.00 \\
\hline & 5 & a0ef7aa8c7200a711f30101de786e2450f7a7f1e884a44831aba30c77f46b478 & 122 & 47.65 \\
\hline & 6 & bbf12b6acb919c42edb035fe0945b414bf0809b666bbb536976139bee4ea9bdd & 124 & 48.43 \\
\hline & Average & & 123.8 & 48.35 \\
\hline
\end{tabular}

Table 10: Sensitivity of hash value to the message for the proposed structures with $M P$ output scheme

\begin{tabular}{cccc}
\hline & Output scheme & $\boldsymbol{B}_{\boldsymbol{i}}$ & $\boldsymbol{H D}_{\boldsymbol{i}} \boldsymbol{\%}$ \\
\hline Structure 1 & $M M O$ & 126.2 & 49.29 \\
& $M M M O$ & 128.6 & 50.23 \\
& $M P$ & 135.40 & 52.89 \\
\hline \hline Structure 2 & $M M O$ & 131.4 & 51.32 \\
r = 8 & $M M M O$ & 126.8 & 49.53 \\
& $M P$ & 135.2 & 52.81 \\
\hline Structure 2 & $M M O$ & 129.6 & 50.62 \\
$\mathbf{r}=\mathbf{2 4}$ & $M M M O$ & 124.8 & 48.75 \\
& $M P$ & 123.8 & 48.35 \\
\hline
\end{tabular}

Table 11: A comparison of average $B_{i}$ and $H D_{i}(\%)$ for message sensitivity

\begin{tabular}{|c|c|c|c|c|}
\hline & Message variants & Hexadecimal hash values & $B_{i}$ & $H D_{i} \%$ \\
\hline \multirow[t]{6}{*}{ Structure 1} & 1 & bedf7967520105d114e2cdf3399f52394a53e276bb104307345bacf93e317ef6 & & \\
\hline & 2 & 60f63ae88faea074964bc5e71022d77003f61ed4dddd8b027c7826e8f31725ff & 116 & 45.31 \\
\hline & 3 & 3e7a24001b11a0a5376d55d073e5910e1bb3b98e4736793ca8bcdf4b5da27b41 & 127 & 49.60 \\
\hline & 4 & fd8fe49f2c5013871f1e291d6c74ceefeb9c4eead9a236d6b923bb04da3c7f4b & 135 & 52.73 \\
\hline & 5 & 054c289004f47fde2fd041e5e830cd4a74d9b586ba2b79835fb5ee13c7289717 & 139 & 54.29 \\
\hline & Average & - & 129.25 & 50.48 \\
\hline \multirow{6}{*}{$\begin{array}{c}\text { Structure } 2 \\
r=8\end{array}$} & $\overline{1}$ & 1/d6238873699dd1c252e02c88e1d2a380d9b5ea8e6c09c788fa4d3955b959975 & & \\
\hline & 2 & aab2bfb971b64b4349a5045d277421df6ee299dc209b0bf0ce9bfccff8bbbe $8 \mathrm{~b}$ & 138 & 53.90 \\
\hline & 3 & c5667f505bcb289ec52be2fce9a168b72ad0de3fae396b7654f34cf419309b0f & 123 & 48.04 \\
\hline & 4 & 54b21e25c1ee818897c54e84eca15d2ddbd7b505ef81ba2c099a5c852db33b51 & 121 & 47.26 \\
\hline & 5 & f6e6702867e3c3ee86a4d86a6153b1266f58847a704665417fbc66fc39d8179f & 132 & 51.56 \\
\hline & Average & 200 & 128.5 & 50.19 \\
\hline \multirow{6}{*}{$\begin{array}{c}\text { Structure } 2 \\
\mathrm{r}=\mathbf{2 4}\end{array}$} & 1 & af5e7ca7c83a72c77f0e9b7d47df11b0f66cadc862d6f522d592dc5ad9bae938 & & \\
\hline & 2 & 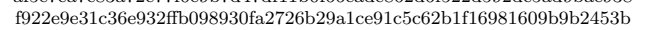 & 125 & 48.82 \\
\hline & 3 & 3566ab26fff9c3a232368b624267c3397ab1099ba744ff5f6ec97a7cbc483fa5 & 126 & 49.21 \\
\hline & 4 & 3b6a773dfe06e246ab3f53c3c9a0af08123346bb8a0e58a17caf6046992e08a7 & 130 & 50.78 \\
\hline & 5 & 40ed183aa3cfb41d9d6f7e304d9ab05a0007044b0db84f039f4315c046051641 & 146 & 57.03 \\
\hline & Average & & 131.75 & 51.46 \\
\hline
\end{tabular}

Table 12: Sensitivity of hash value to the secret key for the proposed structures with $M M O$ output scheme

In Tables 12, 13, and 14, we present the obtained results of $h_{i}, B_{i}$, and $H D_{i}(\%)$ under each condition for the two proposed structures with their output schemes, i.e., $M M O, M M M O$, and $M P$.

In Table 15, we reassessed the obtained results and even for a single test, the results are inside the normal range. Therefore, the proposed hash functions have high key sensitivity. 


\begin{tabular}{|c|c|c|c|c|}
\hline & Message variants & Hexadecimal hash values & $B_{i}$ & $H D_{i} \%$ \\
\hline \multirow{6}{*}{ Structure 1} & 1 & 719adf0e0cdf5b149edc54efdbc09bb6df5a0ce3d3ac9bccc39ac5a64ea65531 & & \\
\hline & 2 & f2d $4772 \mathrm{a} 5 \mathrm{a} 605 \mathrm{c} 729 \mathrm{e} 8 \mathrm{ad} 2 \mathrm{c} 3 \mathrm{db} 016 \mathrm{a} 20135 \mathrm{f} 617 \mathrm{~b} 98 \mathrm{c} 4366 \mathrm{bb} 9 \mathrm{~b} 44 \mathrm{cea} 418 \mathrm{afe} 92$ & 114 & 44.53 \\
\hline & 3 & 23c5a8b268979416f80a32c7aa272c23cd293e20fe3547f8a621815276b3ebab & 130 & 50.78 \\
\hline & 4 & 75c848fa05415217403dbc2235da6d8fa7fa18b7526b376e4fbb89497303c340 & 120 & 46.87 \\
\hline & 5 & 22c9b90204e4522181389ccff6ab7d24547415b87c8cbd3425c83929c3221024 & 118 & 46.09 \\
\hline & Average & - & 120.50 & 47.07 \\
\hline \multirow{6}{*}{$\begin{array}{c}\text { Structure } 2 \\
r=8\end{array}$} & 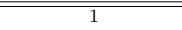 & a594a994aa162adca654e889dea0e6344190aa02328302465570df8f0084f5e6 & & \\
\hline & 2 & 96ebc3ab71912e96b77b6c0db2ad2b0b300484abec4c326bbf10e7b5263ba545 & 127 & 49.60 \\
\hline & 3 & 67d10bee9dedd7e06d58ee10aca74ca3336000f1984a54591d4f9e33face2a1a & 138 & 53.90 \\
\hline & 4 & d2db99f2d01e0b5933c37fd86f8983577893b03f490abe2683e2e11870d1df69 & 123 & 48.04 \\
\hline & 5 & 6d5b61d74e75cd983b4fobf3913211dd991aa35f378842bb187d734f708a49db & 126 & 49.21 \\
\hline & Average & 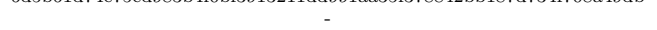 & 128.5 & 50.19 \\
\hline \multirow{6}{*}{$\begin{array}{c}\text { Structure } 2 \\
\mathrm{r}=\mathbf{2 4}\end{array}$} & 1 & 6abbd825d6b17184a5fc558670f9f78d91b3812c899c8a062ef855507b4a81e5 & & \\
\hline & 2 & 8741188aadde9edba0310e69541c85936202a4c7ef4de93e9906bdd 970931948 & 149 & 58.20 \\
\hline & 3 & e10308d6126ebaef0ed5982b03e0c27a521060a570aa0a2cf692e63d2d149336 & 137 & 53.51 \\
\hline & 4 & e8818d36b227e849ed6e3a121745f8d8803bf9425384745fba6a2b1b7adbe32c & 119 & 46.48 \\
\hline & 5 & 26354fobc5a4e6385ac23c715acccf65c2d2b28785e504a4a2966f21189b8fde & 132 & 51.56 \\
\hline & Average & - & 134.25 & 52.44 \\
\hline
\end{tabular}

Table 13: Sensitivity of hash value to the secret key for the proposed structures with $M M M O$ output scheme

\begin{tabular}{|c|c|c|c|c|}
\hline & Message variants & Hexadecimal hash values & $B_{i}$ & $H D_{i} \%$ \\
\hline \multirow[t]{6}{*}{ Structure 1} & 1 & a005e50f9673ecee6e80c07c550e53f8a950cb4a91176a2a340b5822ec2f28c4 & & \\
\hline & 2 & 27de6d91694c777474b94f2a4ec3ed8c5b5b0da8c38fed5b4c75e2e2bf97972f & 143 & 55.85 \\
\hline & 3 & 3fa8a997b46131a1429d0006b6c03f181898632313a64f3da8143d1cadd66925 & 122 & 47.65 \\
\hline & 4 & f670f60cfc1daecb0c81988735b736c8c18851cebe5b94a6f1234f49bd4d5209 & 117 & 45.70 \\
\hline & 5 & 7c68bc63287bfe02badbceb99cdde6a0ef5e9e7429d1dc3d2a9bf90b34a6402c & 123 & 48.04 \\
\hline & Average & 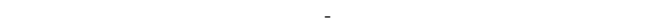 & 126.25 & 49.31 \\
\hline \multirow{6}{*}{$\begin{array}{c}\text { Structure } 2 \\
r=8\end{array}$} & 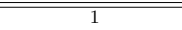 & $\begin{array}{l}31882869 \text { cce69d7734f0078d29f297841b99d3f9786a1cf522 } \\
\end{array}$ & 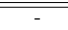 & \\
\hline & 2 & 0b840b10ffda4c9feb4dabf4ab2f642ffe55f730386b8d295534368af526fa33 & 136 & 53.12 \\
\hline & 3 & 2f65ed46a3cb9b0ebb1cf7cd52558de58e2ebc7474b01f169a6b30067e20e5a5 & 134 & 52.34 \\
\hline & 4 & cf524afe65de3a8123e43e61540a28180f0be21669a3ca4b4d62fdca34f538b5 & 139 & 54.29 \\
\hline & 5 & 27d7a12c3a95c9f52148b43d60c7dbd3acd0b774c885d712bf2bb7673b77443e & 131 & 51.17 \\
\hline & Average & - & 135 & 52.73 \\
\hline \multirow{6}{*}{$\begin{array}{c}\text { Structure } 2 \\
\mathrm{r}=\mathbf{2 4}\end{array}$} & 1 & a86e4c2ff1450a08a173b2d9ef27d941fcb9a06f76ad1e70108192ce3cd02a16 & & \\
\hline & 2 & 37235dea611e13421ca8545078d0ec3a88654cfbc4e24bd64dd110ce2ed4ea3e & 121 & 47.26 \\
\hline & 3 & 7f60df23e3570ba37890a0b199e891835757fabc67b96e2cbbd02d0f64629cb7 & 120 & 46.87 \\
\hline & 4 & $5581 \mathrm{e}$ & 127 & 49.60 \\
\hline & 5 & 149bb7e22e3a018254a5cfb711e192471971857c96663e6ec189762548f09ca3 & 139 & 54.29 \\
\hline & Average & & 126.75 & 49.51 \\
\hline
\end{tabular}

Table 14: Sensitivity of hash value to the secret key for the proposed structures with $M P$ output scheme

\begin{tabular}{cccc}
\hline & Output scheme & $\boldsymbol{B}_{\boldsymbol{i}}$ & $\boldsymbol{H D}_{\boldsymbol{i}} \mathbf{\%}$ \\
\hline Structure 1 & $M M O$ & 129.25 & 50.48 \\
& $M M M O$ & 120.50 & 47.07 \\
& $M P$ & 126.25 & 49.31 \\
\hline \hline Structure 2 & $M M O$ & 128.5 & 50.19 \\
$\mathbf{r}=\mathbf{8}$ & $M M M O$ & 128.5 & 50.19 \\
& $M P$ & 135 & 52.73 \\
\hline Structure 2 & $M M O$ & 131.75 & 51.46 \\
$\mathbf{r}=\mathbf{2 4}$ & $M M M O$ & 134.25 & 52.44 \\
& $M P$ & 126.75 & 49.51 \\
\hline
\end{tabular}

Table 15: A comparison of average $B_{i}$ and $H D_{i}(\%)$ for key sensitivity

\subsubsection{Statistical analysis of diffusion effect}

Since confusion and diffusion were first proposed by Shannon [4] in 1949, they have been extensively used to evaluate the security of cryptographic primitives. In the context of hash functions, confusion is defined as the complexity of the relation between the secret key $K$ and the hash value $h$ for a given message $M$, whereas diffusion is defined as the complexity of the relationship between the message $M$ and the hash value $h$ for a given key $K$. The confusion effect is naturally obtained 
in hash functions and it is very strong in chaos-based hash functions, due to the inherent properties of chaos. In cryptographic hash functions, strong diffusion is required. The ideal diffusion effect is obtained when any single bit change in the message causes a change with a $50 \%$ probability for each bit of a hash value (binary format). This is often referred to the avalanche effect in literature [69].

To evaluate the performance of the two proposed structures with different output schemes, i.e., $M M O, M M M O$, and $M P$, we performed the following diffusion test: the previous defined message $M$ is chosen and a hash value $h$ is generated. Next, a bit in the message is randomly selected and toggled and a new hash value is generated. Then, the number of bits changed $B_{i}$ between the two hash values is calculated. This test is performed at J-time, where $\mathrm{J}=512,1024$, and 2048 tests. The six statistical values concerning this test are calculated as follows:

1. Minimum number of bits changed:

$$
B_{\text {min }}=\min \left(\left\{B_{i}\right\}_{i=1, \ldots, J}\right) \text { bits }
$$

2. Maximum number of bits changed:

$$
B_{\max }=\max \left(\left\{B_{i}\right\}_{i=1, \ldots, J}\right) \text { bits }
$$

3. Mean number of bits changed:

$$
\bar{B}=\frac{1}{J} \sum_{i=1}^{J} B_{i} \text { bits }
$$

4. Mean changed probability (mean of $H D_{i}(\%)$ ) $P=\left(\frac{\bar{B}}{256}\right) \times 100 \%$

5. Standard variance of the changed bit number:

$$
\Delta B=\sqrt{\frac{1}{J-1} \sum_{i=1}^{J}\left(B_{i}-\bar{B}\right)^{2}}
$$

6. Standard variance of the changed probability:

$$
\Delta P=\sqrt{\frac{1}{J-1} \sum_{i=1}^{J}\left(\frac{B_{i}}{256}-P\right)^{2}} \times 100 \%
$$

The obtained statistical results of diffusion presented in Table 16 with 2048 tests demonstrates that the diffusion effect is close to the expected one. Indeed, irrespective of the used structure and the output schemes, both $\bar{B}$ and $P$ are very close to the ideal values (128 bits and $50 \%$, respectively), while $\Delta B$ and $\Delta P$ are very low, which indicates that the diffusion is extremely stable. These results, presented in Table 17, are also confirmed through the tests with $\mathrm{J}=512$ and 1024 , for Structures $\mathbf{1}$ and $\mathbf{2}$ with $M P$ output scheme.

In addition, we draw the histogram $B_{i}$ (Fig. 17) of Structure 1 with $M P$ output scheme to show that the values of $B_{i}$ are centered on the ideal value 128 bits. Similar results are obtained for the other proposed hash functions as well.

\subsection{Cryptanalysis}

The attackers make use of some general attack methods that are available to them, which can be applied to any Unkeyed or Keyed hash functions (Fig. 15). These attacks depend only on the hash value length $u$ for the unkeyed hash function and on the hash value length $u$ and the secret key length $|K|$ for the keyed hash function. If the cryptanalyst can find a method to retrieve $K$, the system is entirely compromised (during the key life time) [54,70]. 


\begin{tabular}{ccccc}
\hline & & \multicolumn{3}{c}{ Output schemes } \\
\cline { 3 - 5 } Structure 1 & $B_{\min }$ & 98 & 98 & 100 \\
& $B_{\max }$ & 158 & 158 & 154 \\
& $\bar{B}$ & 127.98 & 127.90 & 127.95 \\
& $P$ & 49.99 & 49.96 & 49.98 \\
& $\Delta B$ & 8.01 & 8.12 & 8.03 \\
& $\Delta P$ & 3.13 & 3.17 & 3.13 \\
\hline \hline Structure 2 & $B_{\min }$ & 99 & 98 & 103 \\
r = 8 & $B_{\max }$ & 157 & 154 & 157 \\
& $\bar{B}$ & 128.31 & 128.18 & 127.97 \\
& $P$ & 50.12 & 50.07 & 49.99 \\
& $\Delta B$ & 8.03 & 8.17 & 8.01 \\
& $\Delta P$ & 3.13 & 3.19 & 3.13 \\
\hline Structure 2 & $B_{\min }$ & 101 & 103 & 100 \\
r $=\mathbf{2 4}$ & $B_{\max }$ & 155 & 156 & 157 \\
& $\bar{B}$ & 127.81 & 127.70 & 127.88 \\
& $P$ & 49.92 & 49.88 & 49.95 \\
& $\Delta B$ & 8.23 & 8.06 & 7.94 \\
& $\Delta P$ & 3.21 & 3.15 & 3.10 \\
\hline
\end{tabular}

Table 16: Diffusion statistical-results for the two proposed structures

\begin{tabular}{ccccc}
\hline & & \multicolumn{3}{c}{ Number of tests } \\
\cline { 3 - 5 } Structure 1 & & 512 & 1024 & 2048 \\
& $B_{\min }$ & 100 & 100 & 100 \\
& $B_{\max }$ & 149 & 152 & 154 \\
& $\bar{B}$ & 128.11 & 128.22 & 127.95 \\
& $P$ & 50.04 & 50.08 & 49.98 \\
& $\Delta B$ & 8.11 & 8.17 & 8.03 \\
& $\Delta P$ & 3.16 & 3.19 & 3.13 \\
\hline \hline Structure 2 & $B_{\min }$ & 104 & 104 & 103 \\
r = 8 & $B_{\max }$ & 150 & 151 & 157 \\
& $\bar{B}$ & 127.98 & 127.88 & 127.97 \\
& $P$ & 49.99 & 49.95 & 49.99 \\
& $\Delta B$ & 7.92 & 7.98 & 8.01 \\
& $\Delta P$ & 3.09 & 3.12 & 3.13 \\
\hline Structure 2 & $B_{\min }$ & 100 & 100 & 100 \\
r $=\mathbf{2 4}$ & $B_{\max } a$ & 153 & 153 & 157 \\
& $\bar{B}$ & 127.85 & 127.96 & 127.88 \\
& $P$ & 49.95 & 49.98 & 49.95 \\
& $\Delta B$ & 8.22 & 8.10 & 7.94 \\
& $\Delta P$ & 3.21 & 3.16 & 3.10 \\
\hline & & & &
\end{tabular}

Table 17: Diffusion statistical-results for the two proposed structures with $M P$ output scheme

\subsubsection{Brute force attacks}

A brute-force attack on a keyed hash function is more difficult than a brute-force attack on an unkeyed hash function. There are two possible types of attacks, which are as follows:

1. Attacks on the hash value $h$, namely Preimage attack, second preimage attack, and collision resistance attack.

2. Attack on the secret key $K$, namely Exhaustive key search attack. 


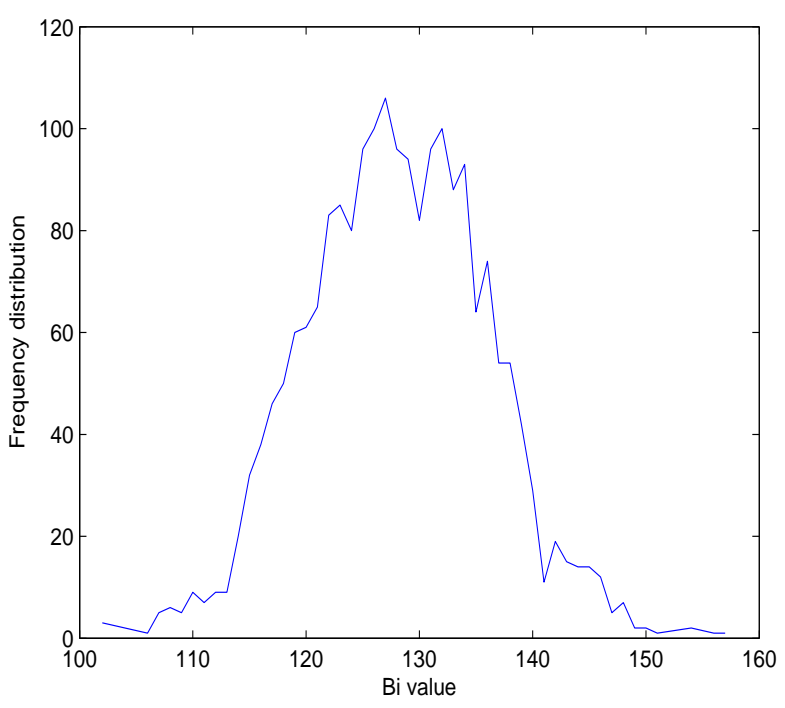

Fig. 17: Histogram of $B_{i}$

For the first type of attacks, for a given secret key $K$, the fastest way to compute a first or second preimages and collision resistance is through a brute force attack that consists of randomly selecting values of $M$ and try each value until a collision occurs. For exhaustive key search attack, the attacker requires known \{message, hash\} pairs.

Preimage and second preimage attacks [71]: In a preimage attack, given only the hash value $h$, the attacker tries to find the original message $M$ in a way such that $H(M)=h$ without attempting to recover the secret key $K$. For example, in an authentication security service, a website stores \{username, $H$ (password) $\}$ in its database instead of \{username, password\}. When a user tries to access the website in question, the website verifies the authenticity of the user by comparing $H$ (input) with the stored hash $H$ (password) (Fig. 18). Now, suppose this database is compromised and an attacker succeeds in accessing a given hash value, then he can try to generate the corresponding message using a preimage attack.

In a second preimage attack, the adversary has more information. Specifically, he knows the hash value $h$ for a given message $M$ and he tries to find another message $M^{\prime}$ that produces the same hash value $h$. For example, in digital signature scheme for data integrity security service, the attacker has access to both document $M$ and its hash $h$ and tries to find a new document $M^{\prime}$, such that $H\left(M^{\prime}\right)=h$, so that he can send the signed new document $M^{\prime}$ as the original signed document $M$ (Fig. 19).

For the first and second preimage attacks, the adversary would have to try, on average, $2^{u-1}$ values of $M$ to find one that generates the given hash value $h$. Our proposed structures produce hash values of length 256 bits, so that the minimum amount of work required by an attacker to violate the preimage or second preimage 


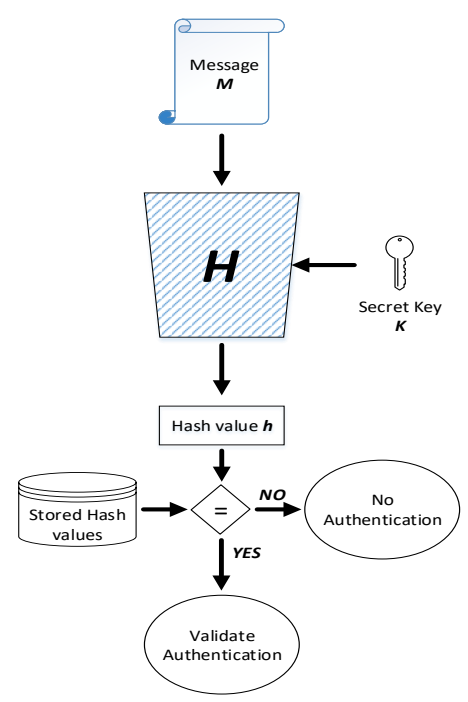

Fig. 18: General scheme of hash authentication

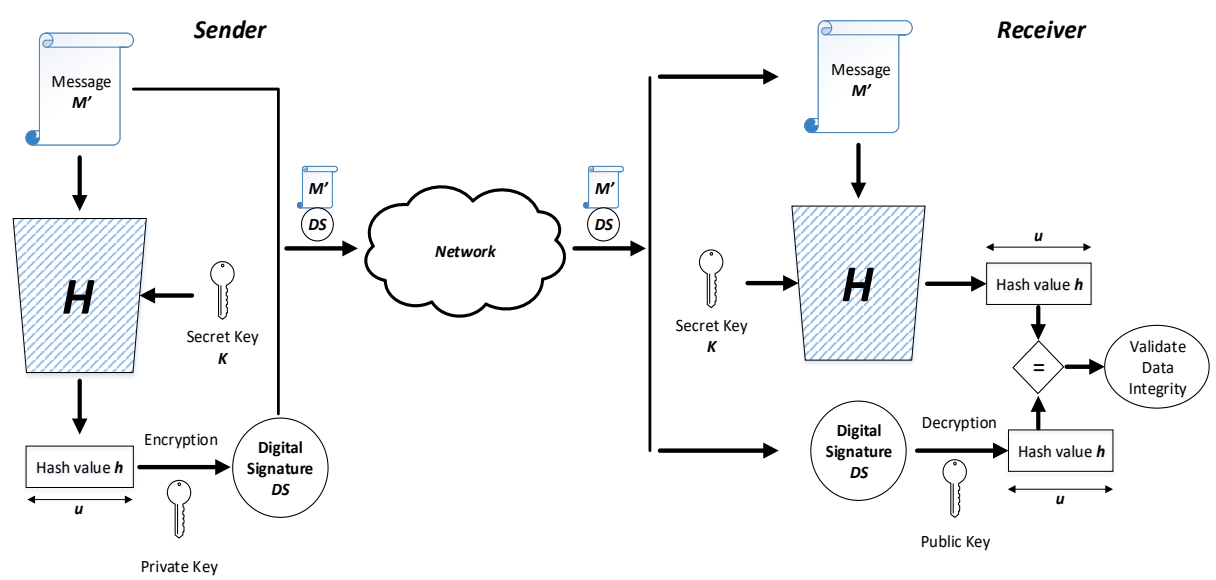

Fig. 19: Second preimage attack on Digital Signature scheme

resistance property should be $2^{256-1}$ operations, which is considered very high. Thus, the proposed hash functions are robust against first and second preimage attacks.

Collision resistance attack (Birthday attack) [72]: In the collision resistance attack, the attacker tries to find two messages $\left(M, M^{\prime}\right)$ that collide with the same hash value $h$. The minimum amount of work required by an attacker to violate the collision resistance property is approximately $2^{u / 2}$ operations. This required effort 
is proven by a mathematical result referred to as the birthday paradox, which is detailed in the example below.

Let us take the situation whether any two students in a class have the same birthday. Suppose that the class has 23 students. If a teacher specifies a day (say August 11), then the probability that at least one student has the same birthday as any other student is $\left(1-\frac{(365 \times 364 \times \ldots \times 343)}{365^{(23)}}\right)=50.73 \%$. Birthday attack is widely exploited for finding any two messages $M$ and $M^{\prime}$, such that $H(M)=H\left(M^{\prime}\right)$, then the couple $\left(M, M^{\prime}\right)$ is named a collision. If the length of $h$ is $u$ and hash values are random with a uniform distribution, an adversary can expect to find a collision $\left(M, M^{\prime}\right)$ with a $50 \%$ probability within $\sqrt{2^{u}}=2^{u / 2}$ attempts. Yuval [73] proposed the following strategy in $D S$ application (Fig. 19) to exploit the birthday paradox in a collision resistant attack without attempting to recover the secret key $K$ :

1. The sender is prepared to sign a legitimate message $M$ by appending the appropriate ciphered $u$-bit hash code using its private key.

2. The attacker generates $2^{u / 2}$ minor variations $\delta M$ of the message $M$, where all of them essentially convey the same meaning along with storing these messages and their hash values in a table.

3. The attacker tries to find a fraudulent message $M^{\prime}$ that has the same sender's signature which was generated using the second preimage attack.

4. The attacker generates $2^{u / 2}$ minor variations $\delta M^{\prime}$ of $M^{\prime}$, where all of them essentially convey the same meaning. For each $\delta M^{\prime}$, the attacker computes $H\left(\delta M^{\prime}\right)$, checks for matches with any of the $H(\delta M)$ values, and continues until a match is found, $H\left(\delta M^{\prime}\right)=H(\delta M)$.

5. Then, the attacker gives the valid fraudulent message $\delta M^{\prime}$ to the sender for signature and this signature can then be attached to the fraudulent message for transmission to the intended receiver. Thus, the attacker is assured of success even though the encryption key is not known.

Another practical example is when the attacker finds a collision between a valid Microsoft Windows security patch and a malware. Then, the attacker sends his malware to sign it, in any certificate company, and ship it to Microsoft Windows users around the world. Later, when a user tries to download the new patch, his computer gets infected.

Also, for collision resistance attack, the length of hash value $h$ determines the security and the proposed hash functions are secure against these kinds of attacks because an attacker needs, on average, $2^{128-1}$ tries.

Exhaustive key search attack [68,74]: In keyed $C N N$ hash functions, if the attacker has access to a pair (message, digest), then normally the key can be found by exhaustive searching and, on average, the attacker needs $2^{|K|-1}$ tries, where $|K|$ is the length of the secret key $K$. Thus, the level of effort for brute force attack on keyed hash functions can be expressed as $\min \left(2^{|K|}, 2^{u}\right)$. As $|K|=160$ bits, consequently, the proposed hash functions are immune against these kinds of attacks.

\subsubsection{Cryptanalytical attacks}

Cryptanalytic attacks seek to exploit some properties of the keyed hash function to perform some attacks other than brute force attacks. An ideal keyed hash function 
should require a cryptanalytic effort greater than or equal to the brute force effort. Far less research has been conducted on developing such attacks. A useful survey of some methods for specific keyed hash functions is developed in [75]. In the following paragraphs, we apply the main cryptanalytic attacks of the literature on the proposed hash functions, which are listed below:

1. Length extension attack (Padding attack)

2. Meet-in-the-middle preimage attack

3. Joux attack (Multi-collision attack)

4. Long message second preimage attack

5. Herding attack

Length extension attack [76,77]: In cryptography and computer security, a length extension attack is a type of attack where an attacker can use $H(M)$ and the length of $M$ to calculate $H(M \| E M)$ for an attacker-controlled extended message $E M$. The following attack is applied on Merkle - Damgard structure that is transformed on keyed hash functions by adding the secret key $K$ in the beginning of the message $M(M A C)$. This attack allows the inclusion of extra message $(E M)$ into a signed message, but needs to know the length of secret key $K$. Algorithms like $M D 5, S H A-1$, and $S H A-2$ that are based on the Merkle-Damgard construction are vulnerable to these kinds of attacks. However, $H M A C$ is not vulnerable to the length extension attacks [78].

The attacker can perform the following steps. Suppose Alice sends (message $M$, hash value $h$ ) as a pair to Bob. Let us assume that the attacker has access to the message and its hash, then, he can easily calculate, from this pair, a new hash value $h^{\prime}$, which is as follows:

1. Pad the message $M$ with an arbitrary extended message $E M$ with a length equal or multiple of a size block.

2. Set the digest $h$ as the secret key.

3. Calculate the new hash value $h^{\prime}$ corresponding to $(M \| E M)$. This means that $h$ is used as the key for the added block(s) of $(M \| E M)$.

4. Substitute $(M, h)$ pair by $\left(M \| E M, h^{\prime}\right)$ and send it to $B o b$ as a valid signature (Fig. 20).

In our proposed hash functions, the secret key $K$ is not pre-pended to the message $M$ but used as an input for the Chaotic System to produce the necessary supplies to $C N N$. Then, such an attack can not be conducted.

Meet-in-the-middle preimage attack (MITM) [79,80]: The meet-in-the-middle preimage attack is a generic cryptanalytic approach that is originally applied to the cryptographic systems based on block ciphers (Chosen plain-text attack). In 2008, Aoki and Sasaki [80] noticed that the MITM attack could be applied to hash functions, to find preimage, second preimage, or collision for intermediate hash chaining values instead of the hash value $h$. This attack has successfully broken several designs: the $M D$ hash family includes $M D 5$ [81], round-reduced $S H A-0$, and $S H A-1$ [80], round-reduced SHA-2 [82], some Davies-Meyer hash constructions, e.g., Tiger [83], reduced HAS-160 [84] and HAVAL [85]. The steps of MITM attack, illustrated in Fig. 21 for a given secret key $K$, can be explained as follows: 


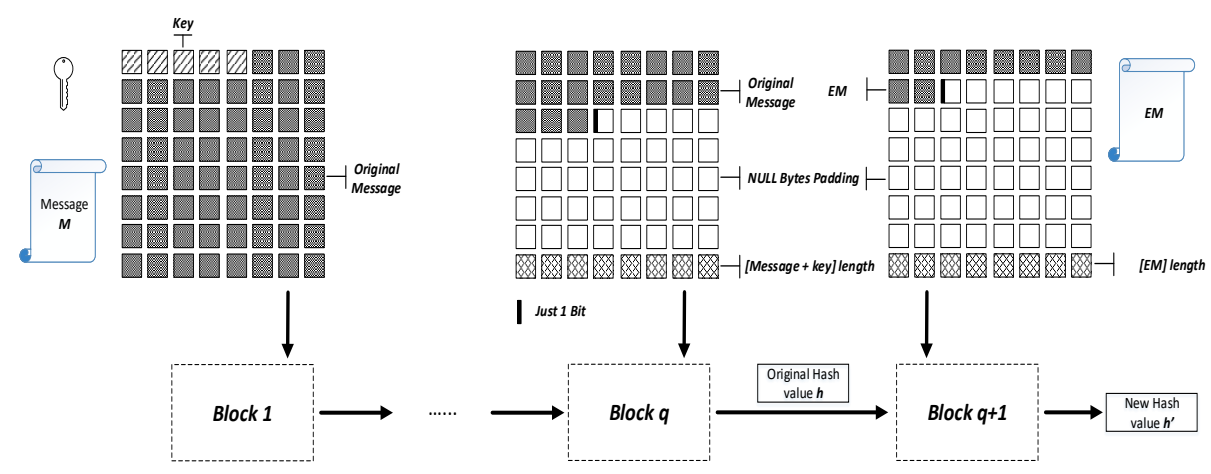

Fig. 20: Hash length extension attack

1. Use the hash function $H$ to calculate the hash value $h$ of a message $M$ that is divided into $q$ fixed-size blocks.

2. Split the chain hash function in two parts, where the first part includes $q$-2 blocks and the second part includes the last two blocks $q-1$ and $q$.

3 . Choose a message $Q$ of length $q$ - 2 in the form $\left\{Q_{1}, Q_{2}, \ldots, Q_{q-2}\right\}$.

4. Compute the hash value $K Q_{q-2}$ of the chosen message using $H$.

5. Generate $2^{u / 2}$ random blocks $B_{X}$. For each generated block $B_{X_{i}}$ (instead of $M_{q-1}$ ), start computing (from the splitting point) to generate the chaining hash value: $K Q_{q-1, i}=C\left(B_{X_{i}}, K Q_{q-2}\right), i=1,2, \ldots, 2^{u / 2}$, which forms a list $L_{B_{X}}$ containing all the computed chaining values $\left(K Q_{q-1, i}\right)_{X}, i=1,2$, $\ldots, 2^{u / 2}$ at the matching point.

6. Generate $2^{u / 4}$ random blocks $B_{Y}$. For each generated block $B_{Y_{j}}, j=1, \ldots, 2^{u / 4}$ (instead of $\left.M_{q}\right)$, start calculating $K Q_{q, k}\left(k=1,2, \ldots, 2^{u / 4}\right)$ with $K Q_{q, k}=$ $C\left(B_{Y_{j}}, K Q_{q-1, k}\right)\left(k=1,2, \ldots, 2^{u / 4}\right)$. Then form a list $L_{B_{Y_{j, k}}}$ containing the chaining values of $\left(K Q_{q-1, j, k}\right)_{Y}\left(k=1,2, \ldots, 2^{u / 4}\right)$. Then, $L_{B_{Y}}$ is compared to $L_{B_{X}}$ to find a collision at the matching point.

7. If a collision is found, then form the message $\left\{Q_{1}, Q_{2}, \ldots, Q_{q-2}, B_{X_{i}}, B_{Y_{j}}\right\}$ that gives the desired hash value $h$ and, therefore, use it to produce the same digital signature. Otherwise, repeat the above six steps with a different chosen message $\left\{Q_{1}, Q_{2}, \ldots\right.$, $\left.Q_{q-2}\right\}$.

The probability that one element $\left\{K Q_{q-1, j, k}\right\}_{Y}$ from $L_{B_{Y}}$ matches one element $\left\{K Q_{q-1, k}\right\}_{X}$ from $L_{B_{X}}$ is equal to $\frac{1}{2^{u / 2}}$. Otherwise, the probability is $\left(1-\frac{1}{2^{u / 2}}\right)$. For all the elements of $L_{B_{Y}}$, the probability that none of them are equal to an element of $B_{X}$, is $\left(1-\frac{1}{2^{u / 2}}\right)^{2^{u / 2}}$. Given that, $(1-x) \leq e^{-x}$, the previous expression can be approximated by: $\left(e^{-1 / 2^{\frac{u}{2}}}\right)^{2^{u / 2}}=e^{-1}$. Then, the probability that one intermediate matching value occurs is:

$$
P=1-e^{-1}=0.632
$$




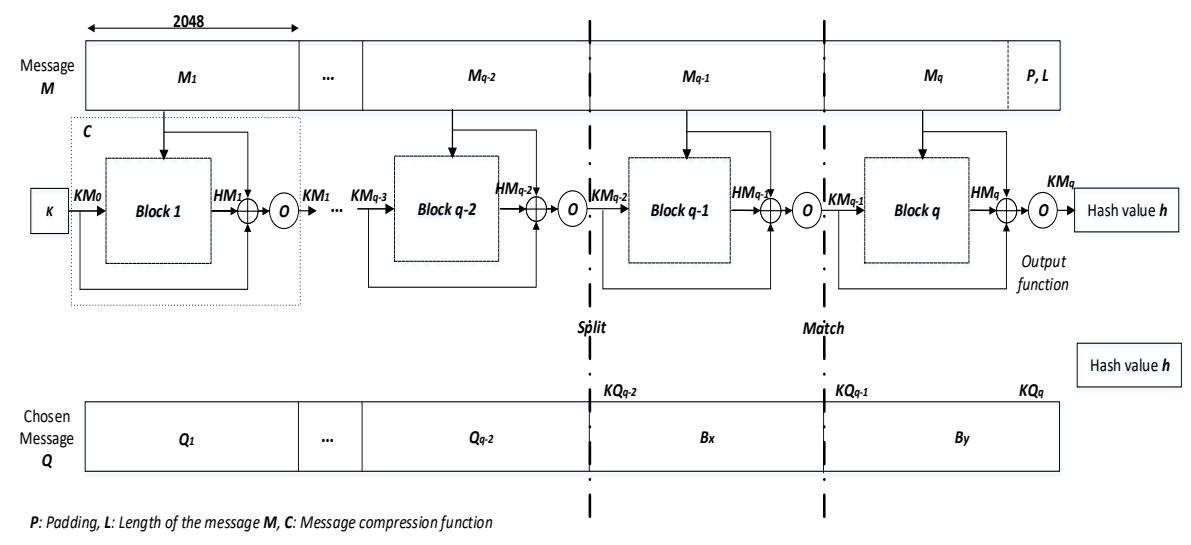

Fig. 21: Meet-in-the-middle preimage attack

As our hash functions are preimage resistant, the effort to succeed the meet-inthe-middle attack with probability 0.632 is $2^{u / 2}$.

Joux attack [86]: A collision attack takes time of order $2^{u / 2}$ (sec. 4.3.1). A multicollision attack means that a set of messages that all have the same hash value $h$. In 2004, Joux showed that searching multi-collisions is not so hard when it comes to finding ordinary collision. Indeed, he demonstrated that finding $2^{t}$ collisions cost only about $t$ times a single collision attack, $t \times 2^{u / 2}$ instead of $2^{u\left(2^{t}-1\right) / 2^{t}}$ evaluations [54]. To illustrate this relation, let we show how 4 collisions $(t=2)$ can be obtained with only two calls of a collision finding machine. This collision finding machine uses birthday attack algorithm. For a given secret key $K$, a first call to the collision finding machine generates two different blocks $M_{1}$ and $M_{1}^{\prime}$ that yield a collision: $K M_{1}=C\left(M_{1}, K\right)=C\left(M_{1}^{\prime}, K\right)$. Then, a second call to the same collision finding machine locates two other blocks $M_{2}$ and $M_{2}^{\prime}$ such that $C\left(M_{2}, K M_{1}\right)=C\left(M_{2}^{\prime}, K M_{2}\right)$. When putting these two steps together, we obtain the following 4 collisions:

$C\left(M_{2}, C\left(M_{1}, K\right)\right)=C\left(M_{2}^{\prime}, C\left(M_{1}, K\right)\right)$

$=C\left(M_{2}, C\left(M_{1}^{\prime}, K\right)\right)=C\left(M_{2}^{\prime}, C\left(M_{1}^{\prime}, K\right)\right)$.

Joux claimed that this basic idea can be extended to much larger collisions by using more calls to the collision finding machine. More precisely, using $t$ calls, we can build $2^{t}$-collision for a given hash function $H$. All of the $2^{t}$ hashing processes go through $K M_{1}, K M_{2}, \ldots, K M_{t}$. A schematic representation of these $2^{t}$ blocks together with their common intermediate hash values is drawn in Fig. 22.

Furthermore, Joux observed that, for two independent hash functions $H$ and $G$ and a given message $M$ with $H(M)=h$ and $G(M)=g$, the concatenation of the two obtained hash values $(h \| g)$ is not more secure against collision attacks, preimage resistance attack, and second preimage attack than any of the two hash functions taken separately. 


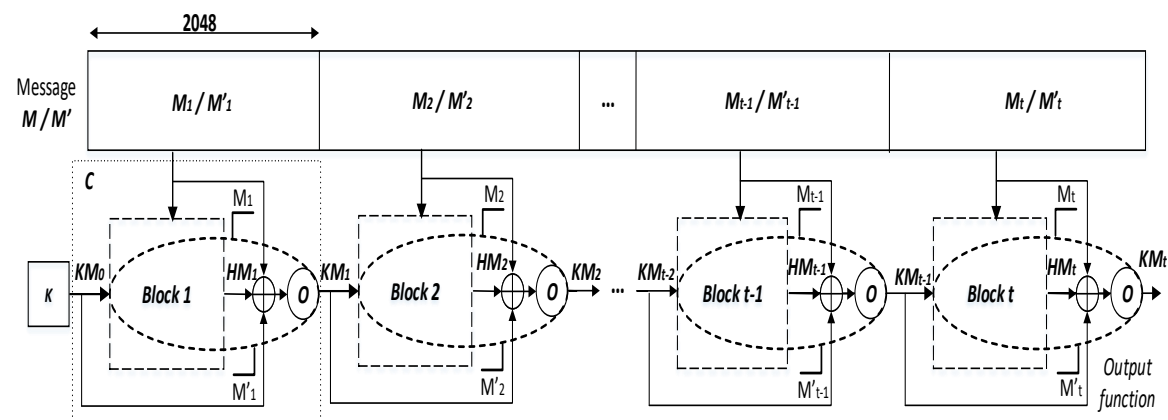

C: Message compressionfunction

Fig. 22: Joux attack

Long message second preimage and Herding attacks The Long message second preimage attack [87] and the Herding attack [88] are closely related to the Joux attack. For the first kind of attack, the attacker can find a second preimage for a message $M$ of $2^{b}$ blocks with $b \times 2^{u / 2+1}+2^{u-b+1}$ effort. For the second attack, the needed work by the attacker to find $2^{t}$ collisions is $2^{u-t-1}+2^{u / 2+t / 2+2}+t \times 2^{u / 2+1}$.

\subsection{Speed analysis}

We evaluated the computing performance of the two proposed hash functions with their output schemes for different message lengths. For this purpose, we calculated the average hashing time $H T$ (micro second), the average hashing throughput $H T H$ (MBytes/second) and the needed number of cycles to hash one Byte $N C p B$ (cycles/Byte).

$$
\begin{gathered}
H T H(\text { MBytes } / s)=\frac{\text { Message size }(\text { MBytes })}{\text { Average hashing time }(s)} \\
N C p B(\text { cycles } / \text { Byte })=\frac{C P U \text { speed }(H z)}{H T H(\text { Byte } / s)}
\end{gathered}
$$

We used a computer with a 2.6 GHZ Intel core i5-4300M CPU with 4 GB of RAM running Ubuntu Linux 14.04.1 (32-bit). In Tables 18, 19, and 20, the average HT, the average $H T H$, and the average $N C p B$ for the two structures with their output schemes are presented. It was observed that, irrespective of the output schemes, the computing performance of Structure $\mathbf{2}$ is approximately twice better than the computing performance of Structure 1, even for $r=24$ rounds. To focus more on these results, the $H T H$ for the two structures with their output schemes 23 were drawn.

The variation of computing performance according to the size of the message is due to the transition phase of both chaotic system and chaotic activation function 


\begin{tabular}{|c|c|c|c|c|c|c|c|c|c|}
\hline \multirow{2}{*}{$\begin{array}{c}\text { Message } \\
\text { length }\end{array}$} & \multicolumn{3}{|c|}{ Structure 1} & \multicolumn{3}{|c|}{ Structure $2-r=8$} & \multicolumn{3}{|c|}{ Structure $2-r=24$} \\
\hline & $H T$ & $H T H$ & $N C p B$ & $H T$ & HTH & $N C p B$ & $H T$ & $H T H$ & $N C p B$ \\
\hline 513 & 8.60 & 57.37 & 43.70 & 4.47 & 112.02 & 22.71 & 6.73 & 73.21 & 34.20 \\
\hline 1024 & 15.24 & 64.98 & 38.75 & 8.18 & 124.18 & 20.79 & 8.02 & 124.17 & 20.30 \\
\hline 2048 & 27.02 & 72.66 & 34.33 & 13.82 & 143.44 & 17.56 & 15.11 & 132.90 & 19.20 \\
\hline 4096 & 51.13 & 76.50 & 32.46 & 25.73 & 153.06 & 16.34 & 26.99 & 146.33 & 17.13 \\
\hline $10^{4}$ & 122.15 & 78.18 & 31.76 & 60.16 & 159.42 & 15.64 & 62.30 & 153.79 & 16.20 \\
\hline $10^{5}$ & 1211.30 & 79.14 & 31.49 & 590.16 & 162.70 & 15.34 & 626.89 & 154.21 & 16.29 \\
\hline $10^{6}$ & 11972.02 & 79.73 & 31.12 & 5910.81 & 162.14 & 15.36 & 6185.43 & 155.61 & 16.08 \\
\hline
\end{tabular}

Table 18: Hashing time, hashing throughput, and the number of cycles per Byte for Structures 1 and $\mathbf{2}$ with $M M O$ output scheme and 2048 random tests

\begin{tabular}{|c|c|c|c|c|c|c|c|c|c|}
\hline \multirow{2}{*}{$\begin{array}{l}\text { Message } \\
\text { length }\end{array}$} & \multicolumn{3}{|c|}{ Structure 1} & \multicolumn{3}{|c|}{ Structure $2-r=8$} & \multicolumn{3}{|c|}{ Structure 2- $\mathbf{r}=\mathbf{2 4}$} \\
\hline & $H T$ & $H T H$ & $N C p B$ & $H T$ & HTH & $N C p B$ & $H T$ & $H T H$ & $N C p B$ \\
\hline 513 & 8.53 & 57.72 & 43.34 & 5.16 & 99.80 & 26.21 & 6.89 & 71.12 & 35.02 \\
\hline 1024 & 15.11 & 65.65 & 38.42 & 7.78 & 127.88 & 19.77 & 8.03 & 124.46 & 20.40 \\
\hline 2048 & 27.21 & 72.30 & 34.56 & 13.47 & 145.78 & 17.11 & 14.32 & 137.94 & 18.19 \\
\hline 4096 & 51.71 & 75.81 & 32.83 & 25.40 & 154.57 & 16.13 & 26.67 & 147.56 & 16.93 \\
\hline $10^{4}$ & 122.50 & 78.05 & 31.85 & 59.71 & 160.27 & 15.52 & 63.25 & 152.32 & 16.44 \\
\hline $10^{5}$ & 1216.68 & 78.70 & 31.63 & 603.15 & 159.79 & 15.68 & 632.82 & 153.17 & 16.45 \\
\hline $10^{6}$ & 11935.23 & 79.97 & 31.03 & 6015.73 & 160.38 & 15.64 & 6272.66 & 153.96 & 16.30 \\
\hline
\end{tabular}

Table 19: Hashing time, hashing throughput, and the number of cycles per Byte for Structures 1 and $\mathbf{2}$ with $M M M O$ output scheme and 2048 random tests

\begin{tabular}{|c|c|c|c|c|c|c|c|c|c|}
\hline \multirow{2}{*}{$\begin{array}{c}\text { Message } \\
\text { length }\end{array}$} & \multicolumn{3}{|c|}{ Structure 1} & \multicolumn{3}{|c|}{ Structure $2-r=8$} & \multicolumn{3}{|c|}{ Structure 2-r $=24$} \\
\hline & $H T$ & $\mathrm{HTH}$ & $\overline{N C p B}$ & $H T$ & $\mathrm{HTH}$ & $N C p B$ & $H T$ & $\mathrm{HTH}$ & $N C p B$ \\
\hline 513 & 8.67 & 57.19 & 44.04 & 4.45 & 111.99 & 22.61 & 6.76 & 73.19 & 34.36 \\
\hline 1024 & 14.77 & 66.84 & 37.55 & 7.72 & 128.94 & 19.62 & 7.94 & 124.42 & 20.19 \\
\hline 2048 & 27.05 & 72.73 & 34.35 & 13.81 & 143.17 & 17.55 & 16.03 & 127.37 & 20.36 \\
\hline 4096 & 51.52 & 76.12 & 32.71 & 27.42 & 145.93 & 17.41 & 28.16 & 141.84 & 17.88 \\
\hline $10^{4}$ & 122.12 & 78.32 & 31.75 & 59.73 & 160.25 & 15.53 & 63.87 & 151.23 & 16.60 \\
\hline $10^{5}$ & 1232.16 & 78.32 & 32.03 & 585.29 & 163.83 & 15.21 & 631.08 & 153.34 & 16.40 \\
\hline $10^{6}$ & 11866.13 & 80.42 & 30.85 & 5864.95 & 163.29 & 15.24 & 6250.05 & 154.55 & 16.25 \\
\hline
\end{tabular}

Table 20: Hashing time, hashing throughput, and the number of cycles per Byte for Structures 1 and $\mathbf{2}$ with MP output scheme and 2048 random tests

of a neuron. Indeed, the cost of the transition phase is approximately equal $2 \times \operatorname{tr}$ $\times 4=240$ Bytes for Structure $1(\operatorname{tr}=30)$ and 160 Bytes for Structure $2(\operatorname{tr}=$ 20 ) in our implementation.

4.5 Performance comparison with other Chaos-based hash functions of literature and standards hash functions

We compared the performance of the proposed hash functions with some hash functions of literature in terms of statistical analysis and $N C p B$. Table 21 presents the comparison with chaos-based hash function in terms of collision resistance for $M P$ output scheme with 2048 tests. As we can see, except $L i$ et al. [30] our obtained results are more close to the expected values. Table 22 , additionally, presents the comparison of statistical results of diffusion. We observed that the obtained results for all cited references are closed to the expected values. It should be noted that besides the two references [34,38], all the other references in Tables 21 and 22 present structures that work with hash value $h=128$ bits. For comparison purposes, we took the $128 L S B$ hash values. 


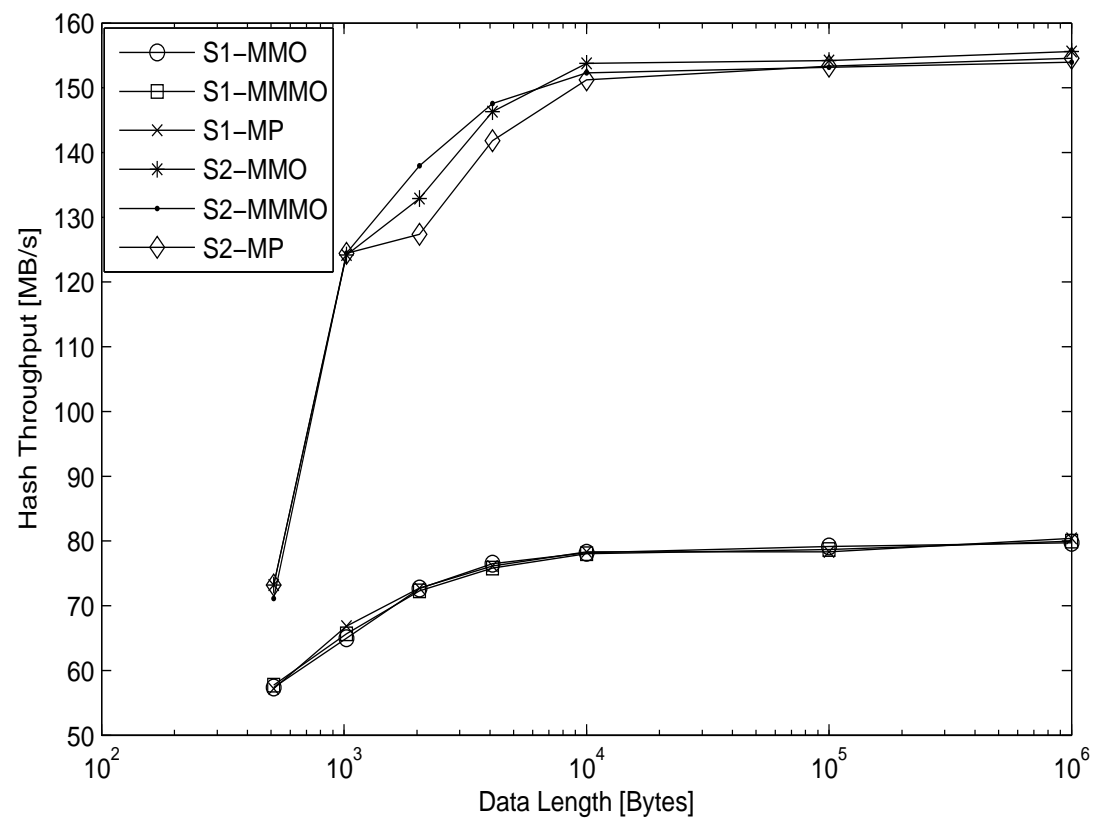

Fig. 23: Comparison of $H T H$ for Structure 1 and Structure $\mathbf{2}-\mathbf{r}=\mathbf{2 4}$ rounds with $M M O, M M M O$, and $M P$ output schemes

Tables 23 and 24 present the comparison of the proposed chaos-based hash functions with standard hash function in terms of collision resistance and diffusion. Aside the values of Structure $2-r=8$ rounds, the obtained results are similar to those obtained by standard hash functions.

The speed performance, in terms of the number of cycles to hash one Byte $(N C p B)$, of the proposed keyed chaos-based hash functions is compared to that of some chaos-based hash functions of literature and with the main standards of the unkeyed and keyed hash functions, which are presented in Tables 25 and 26, respectively. We observed that the $N C p B$ of the Structure $\mathbf{2}$ is approximately twice as fast as the best $N C p B$ obtained by [35], but it is a little bit slower than the SHA-2's $N C p B$ and approximately four times slower than the main keyed hash functions.

\section{Conclusion}

We realized and analyzed the security and computation performance of the two keyed chaotic neural network hash functions, based on Merkle-Dåmgard construction with three output schemes $M M O, M M M O$, and $M P$. The obtained results quantified the robustness of the proposed hash functions for using them in data integrity, message authentication, and digital signature applications. The very good performance is due to the strong one-way property of the combined chaotic system 


\begin{tabular}{|c|c|c|c|c|c|c|c|c|}
\hline \multirow{2}{*}{ Hash function } & \multicolumn{4}{|c|}{ Number of hits $\omega$} & \multicolumn{4}{|c|}{ Absolute difference $d$} \\
\hline & 0 & 1 & 2 & 3 & Mean & Mean/character & Minimum & Maximum \\
\hline Xiao et al. [7] & - & - & - & - & 1506 & 94.12 & 696 & 2221 \\
\hline Xiao et al. [24] & 1926 & 120 & 2 & 0 & 1227.8 & 76.73 & 605 & 1952 \\
\hline Deng et al. [26] & 1940 & 104 & 4 & 0 & 1399.8 & 87.49 & 583 & 2206 \\
\hline Yang et al. [27] & - & - & - & - & - & 93.25 & - & - \\
\hline Xiao et al. [28] & 1915 & 132 & 1 & 0 & 1349.1 & 84.31 & 812 & 2034 \\
\hline Li et al. [13] & 1901 & 146 & 1 & 0 & 1388.9 & 86.81 & 669 & 2228 \\
\hline Wang et al. [16] & 1917 & 126 & 5 & 0 & 1323 & 82.70 & 663 & 2098 \\
\hline Huang [33] & 1932 & 111 & 5 & 0 & 1251.2 & 78.2 & 650 & 1882 \\
\hline Li et al. [29] & 1928 & 118 & 2 & 0 & 1432.1 & 89.51 & 687 & 2220 \\
\hline Li et al. [30] & 1899 & 124 & 25 & 0 & 1367.6 & 85.47 & 514 & 2221 \\
\hline Li et al. [31] & 1920 & 124 & 4 & 0 & 1319.5 & 82.46 & 603 & 2149 \\
\hline He et al. [32] & 1926 & 118 & 4 & 0 & 1504 & 94 & 683 & 2312 \\
\hline Xiao et al. [42] & 1924 & 120 & 4 & 0 & 1431.3 & 89.45 & 658 & 2156 \\
\hline Yu-Ling et al. [89] & 1928 & 117 & 3 & 0 & 1598.6 & 99.91 & 796 & 2418 \\
\hline Xiao et al. [90] & 1932 & 114 & 2 & 0 & 1401.1 & 87.56 & 573 & 2224 \\
\hline Li et al. [91] & 1920 & 122 & 6 & 0 & - & - & - & - \\
\hline Li et al. [92] & 1905 & 135 & 8 & 0 & 1335 & 83.41 & 577 & 2089 \\
\hline Structure 1 & 1931 & 114 & 3 & 0 & 1291.64 & 80.72 & 480 & 2038 \\
\hline Structure 2-r $=8$ & 1929 & 114 & 5 & 0 & 1426.23 & 89.13 & 730 & 2213 \\
\hline Structure $2-r=24$ & 1942 & 106 & 0 & 0 & 1338.85 & 83.67 & 629 & 2071 \\
\hline
\end{tabular}

Table 21: Comparison in terms of collision resistance of the proposed structures with $M P$ output scheme with some chaos-based hash functions

with neural network structure. Indeed, the neuron's activation functions are based on a secure and efficient chaotic generator. Compared to some chaos-based hash functions of literature, the proposed $C N N$ hash functions are more robust and show good results in terms of computation performance. Our future work will focus on the design of the keyed $C N N$ hash function based on Sponge construction, adopted in the standard $S H A-3$, and its Duplex construction for authenticated encryption application.

Conflict of Interest: The authors declare that they have no conflict of interest.

Ethical approval: All procedures performed in studies involving human participants were in accordance with the ethical standards of the institutional and/or national research committee and with the 1964 Helsinki declaration and its later amendments or comparable ethical standards.

Informed consent: Informed consent was obtained from all individual participants included in the study. 


\begin{tabular}{|c|c|c|c|c|c|c|}
\hline Hash function & $B_{m i n}$ & $B_{\max }$ & $\bar{B}$ & $P(\%)$ & $\Delta B$ & $\Delta P \%$ \\
\hline Xiao et al. [7] & - & - & 63.85 & 49.88 & 5.78 & 4.52 \\
\hline Lian et al. [21] & - & - & 63.85 & 49.88 & 5.79 & 4.52 \\
\hline Zhang et al. [19] & 46 & 80 & 63.91 & 49.92 & 5.58 & 4.36 \\
\hline Wang et al. [10] & - & - & 63.98 & 49.98 & 5.53 & 4.33 \\
\hline Xiao et al. [24] & - & - & 64.01 & 50.01 & 5.72 & 4.47 \\
\hline Deng et al. [25] & - & - & 63.91 & 49.92 & 5.58 & 4.36 \\
\hline Deng et al. [26] & - & - & 63.84 & 49.88 & 5.88 & 4.59 \\
\hline Yang et al. [27] & - & - & 64.14 & 50.11 & 5.55 & 4.33 \\
\hline Xiao et al. [28] & - & - & 64.09 & 50.07 & 5.48 & 4.28 \\
\hline Amin et al. [12] & - & - & 63.84 & 49.88 & 5.58 & 4.37 \\
\hline Li et al. [13] & 45 & 81 & 63.88 & 49.90 & 5.37 & 4.20 \\
\hline Wang et al. [16] & - & - & 63.90 & 49.93 & 5.64 & 4.41 \\
\hline Akhavan et al. [17] & 42 & 83 & 63.91 & 49.92 & 5.69 & 4.45 \\
\hline Huang [33] & - & - & 63.88 & 49.91 & 5.75 & 4.50 \\
\hline Li et al. [29] & - & - & 63.80 & 49.84 & 5.75 & 4.49 \\
\hline Wang et al. [11] & 44 & 82 & 64.15 & 50.11 & 5.76 & 4.50 \\
\hline Li et al. [30] & - & - & 63.56 & 49.66 & 7.42 & 5.80 \\
\hline Li et al. [31] & - & - & 63.97 & 49.98 & 5.84 & 4.56 \\
\hline He et al. [32] & 45 & 83 & 64.03 & 50.02 & 5.60 & 4.40 \\
\hline Jiteurtragool et al. [34] & 43 & 81 & 62.84 & 49.09 & 5.63 & 4.40 \\
\hline Teh et al. [35] & - & - & 64.01 & 50.01 & 5.61 & 4.38 \\
\hline Chenaghlu et al. [38] & - & - & 64.12 & 50.09 & 5.63 & 4.41 \\
\hline Akhavan et al. [39] & 43 & 82 & 63.89 & 49.91 & 5.77 & 4.50 \\
\hline Nouri et al. [40] & - & - & 64.08 & 50.06 & 5.72 & 4.72 \\
\hline Xiao et al. [42] & 47 & 83 & 63.92 & 49.94 & 5.62 & 4.39 \\
\hline Yu-Ling et al. [89] & - & - & 64.17 & 50.14 & 5.74 & 4.49 \\
\hline Xiao et al. [90] & - & - & 64.18 & 50.14 & 5.59 & 4.36 \\
\hline Li et al. [91] & - & - & 64.07 & 50.06 & 5.74 & 4.48 \\
\hline Li et al. [92] & - & - & 63.89 & 49.91 & 5.64 & 4.41 \\
\hline Ren et al. [93] & - & - & 63.92 & 49.94 & 5.78 & 4.52 \\
\hline Guo et al. [94] & - & - & 63.40 & 49.53 & 7.13 & 6.35 \\
\hline Yu et al. [95] & 45.6 & 81.8 & 63.98 & 49.98 & 5.73 & 4.47 \\
\hline Zhang et al. [96] & - & - & 64.43 & 49.46 & 5.57 & 4.51 \\
\hline Jiteurtragool et al. [34] & 101 & 153 & 126.75 & 49.51 & 7.98 & 3.12 \\
\hline Chenaghlu et al. [38] & 101 & 168 & 128.08 & 50.03 & 8.12 & 3.21 \\
\hline Structure 1 & 45 & 86 & 64.05 & 50.03 & 5.65 & 4.41 \\
\hline Structure 2-r $=8$ & 42 & 84 & 63.88 & 49.91 & 5.66 & 4.42 \\
\hline Structure 2-r $=24$ & 43 & 85 & 63.90 & 49.92 & 5.60 & 4.37 \\
\hline
\end{tabular}

Table 22: Comparison of the statistical results of diffusion for the proposed structures with $M P$ output scheme with some chaos-based hash functions

\begin{tabular}{lccccccccc} 
Hash function & \multicolumn{3}{c}{ Number of hits $\boldsymbol{\omega}$} & & \multicolumn{5}{c}{ Absolute difference $\boldsymbol{d}$} \\
\cline { 2 - 5 } & 0 & 1 & 2 & 3 & & Mean & Mean/character & Minimum & Maximum \\
\hline SHA2-256 [3] & 1817 & 220 & 11 & 0 & & 2707.10 & 84.59 & 1789 & 3819 \\
Structure 1 & 1803 & 232 & 13 & 0 & & 2633.17 & 82.28 & 1471 & 3779 \\
Structure 2 - $\mathbf{r}=\mathbf{8}$ & 1817 & 215 & 16 & 0 & & 2861.93 & 89.43 & 1707 & 3951 \\
Structure 2 - $\mathbf{r}=\mathbf{2 4}$ & 1815 & 226 & 7 & 0 & & 2615.44 & 81.73 & 1540 & 3671 \\
\hline
\end{tabular}

Table 23: Comparison in terms of collision resistance of the proposed structures with $M P$ output scheme with some chaos-based hash functions 


\begin{tabular}{lcccccc} 
Hash function & $\boldsymbol{B}_{\boldsymbol{m i n}}$ & $\boldsymbol{B}_{\boldsymbol{m a x}}$ & $\overline{\boldsymbol{B}}$ & $\boldsymbol{P}(\boldsymbol{\%})$ & $\boldsymbol{\Delta} \boldsymbol{B}$ & $\boldsymbol{\Delta} \boldsymbol{P} \boldsymbol{\%}$ \\
\hline SHA2-256 [3] & 104 & 154 & 128.01 & 50.00 & 7.94 & 3.10 \\
Structure 1 & 100 & 154 & 127.95 & 49.98 & 8.03 & 3.13 \\
Structure 2 $-\mathbf{r}=\mathbf{8}$ & 103 & 157 & 127.97 & 49.99 & 8.01 & 3.13 \\
Structure 2 $-\mathbf{r}=\mathbf{2 4}$ & 100 & 157 & 127.88 & 49.95 & 7.94 & 3.10 \\
\hline
\end{tabular}

Table 24: Comparison of the statistical results of diffusion for the two proposed structures with MP output scheme and SHA2-256

\begin{tabular}{ccccccccccccc} 
Hash & \multicolumn{3}{c}{ Structure 1 } & \multicolumn{4}{c}{ Structure 2- $\mathbf{r}=\mathbf{8}$} & \multicolumn{2}{c}{ Structure 2- $\mathbf{r}=\mathbf{2 4}$} & Wang [10] & Akhavan [17] & Teh [35] \\
function & $M M O$ & $M M M O$ & $M P$ & $M M O$ & $M M M O$ & $M P$ & $M M O$ & $M M M O$ & $M P$ & & & \\
\hline $\boldsymbol{N} C \boldsymbol{B} \boldsymbol{B}$ & 31.12 & 31.03 & 30.85 & 15.36 & 15.64 & 15.24 & 16.08 & 16.30 & 16.25 & 122.4 & 105.5 & 28.45 \\
\hline
\end{tabular}

Table 25: Comparison of $N C p B$ of the proposed structures with three output schemes with some chaos-based hash functions

\begin{tabular}{|c|c|c|c|c|c|c|c|c|c|c|}
\hline \multirow{2}{*}{ Hash function } & \multicolumn{3}{|c|}{ Structure 1} & \multicolumn{3}{|c|}{ Structure $2-r=8$} & \multicolumn{3}{|c|}{ Structure $2-r=24$} & \multirow{2}{*}{ SHA2-256 } \\
\hline & $M M O$ & $M M M O$ & $M P$ & $M M O$ & $M M M O$ & $M P$ & $M M O$ & $M M M O$ & $M P$ & \\
\hline$N C p B$ & 31.12 & 31.03 & 30.85 & 15.36 & 15.64 & 15.24 & 16.08 & 16.30 & 16.25 & 11.87 \\
\hline $\operatorname{ash} f$ & VMAC & HMAC & GCM & CMAC & DMAC & CBC-M & LAKE 2 & & & \\
\hline$N C p B$ & 0.42 & 14.42 & 0.42 & 4.41 & 4.40 & 2.88 & 2.58 & & & \\
\hline
\end{tabular}

Table 26: Comparison of $N C p B$ of the proposed hash functions with the unkeyed and keyed standards 


\section{References}

1. S. H. Islam, "Provably secure dynamic identity-based three-factor password authentication scheme using extended chaotic maps," Nonlinear Dynamics, vol. 78, no. 3, pp. 2261-2276, 2014.

2. K. Chain and W.-C. Kuo, "A new digital signature scheme based on chaotic maps," Nonlinear dynamics, vol. 74, no. 4, pp. 1003-1012, 2013.

3. S. H. Standard and P. FIPS, "180-2," August, vol. 1, p. 72, 2002.

4. N. SHA, "standard: Permutation-based hash and extendable-output functions," FIPS PUB, vol. 202, p. 2015, 3.

5. K.-W. Wong, "A combined chaotic cryptographic and hashing scheme," Physics letters A, vol. 307, no. 5, pp. 292-298, 2003.

6. H. S. Kwok and W. K. Tang, "A chaos-based cryptographic hash function for message authentication," International Journal of Bifurcation and Chaos, vol. 15, no. 12, pp. 40434050, 2005.

7. D. Xiao, X. Liao, and S. Deng, "One-way hash function construction based on the chaotic map with changeable-parameter," Chaos, Solitons $\&$ Fractals, vol. 24, no. 1, pp. 65-71, 2005.

8. X. Yi, "Hash function based on chaotic tent maps," IEEE Transactions on Circuits and Systems II: Express Briefs, vol. 52, no. 6, pp. 354-357, 2005.

9. G. Arumugam, V. L. Praba, and S. Radhakrishnan, "Study of chaos functions for their suitability in generating message authentication codes," Applied Soft Computing, vol. 7, no. 3, pp. 1064-1071, 2007.

10. Y. Wang, X. Liao, D. Xiao, and K.-W. Wong, "One-way hash function construction based on 2d coupled map lattices," Information Sciences, vol. 178, no. 5, pp. 1391-1406, 2008.

11. Y. Wang, K.-W. Wong, and D. Xiao, "Parallel hash function construction based on coupled map lattices," Communications in Nonlinear Science and Numerical Simulation, vol. 16, no. 7 , pp. 2810-2821, 2011.

12. M. Amin, O. S. Faragallah, and A. A. A. El-Latif, "Chaos-based hash function (cbhf) for cryptographic applications," Chaos, Solitons \&f Fractals, vol. 42, no. 2, pp. 767-772, 2009.

13. Y. Li, D. Xiao, and S. Deng, "Secure hash function based on chaotic tent map with changeable parameter," High Technol. Lett, vol. 18, no. 1, pp. 7-12, 2012.

14. J. Liu, X. Wang, K. Yang, and C. Zhao, "A fast new cryptographic hash function based on integer tent mapping system.," JCP, vol. 7, no. 7, pp. 1671-1680, 2012.

15. M. Maqableh, A. B. Samsudin, and M. A. Alia, "New hash function based on chaos theory (cha-1)," International Journal of Computer Science and Network Security, vol. 8, no. 2, pp. 20-27, 2008.

16. Y. Wang, M. Du, D. Yang, and H. Yang, "One-way hash function construction based on iterating a chaotic map," in Computational Intelligence and Security Workshops, 2007. CISW 200\%. International Conference on, pp. 791-794, IEEE, 2007.

17. A. Akhavan, A. Samsudin, and A. Akhshani, "Hash function based on piecewise nonlinear chaotic map," Chaos, Solitons \& Fractals, vol. 42, no. 2, pp. 1046-1053, 2009.

18. Q.-h. Zhang, H. Zhang, and Z.-h. Li, "One-way hash function construction based on conservative chaotic systems," in Information Assurance and Security, 2009. IAS'09. Fifth International Conference on, vol. 2, pp. 402-405, IEEE, 2009.

19. J. Zhang, X. Wang, and W. Zhang, "Chaotic keyed hash function based on feedforwardfeedback nonlinear digital filter," Physics Letters A, vol. 362, no. 5, pp. 439-448, 2007.

20. D. Xiao and X. Liao, "A combined hash and encryption scheme by chaotic neural network," Advances in Neural Networks-ISNN 2004, pp. 13-28, 2004.

21. S. Lian, J. Sun, and Z. Wang, "Secure hash function based on neural network," Neurocomputing, vol. 69 , no. 16 , pp. 2346-2350, 2006

22. S. Lian, Z. Liu, Z. Ren, and H. Wang, "Hash function based on chaotic neural networks," in Circuits and Systems, 2006. ISCAS 2006. Proceedings. 2006 IEEE International Symposium on, pp. 4-pp, IEEE, 2006.

23. X. Liu and C. Xiu, "Hysteresis modeling based on the hysteretic chaotic neural network," Neural Computing and Applications, vol. 17, no. 5-6, pp. 579-583, 2008.

24. D. Xiao, X. Liao, and Y. Wang, "Parallel keyed hash function construction based on chaotic neural network," Neurocomputing, vol. 72, no. 10, pp. 2288-2296, 2009.

25. S. Deng, D. Xiao, Y. Li, and W. Peng, "A novel combined cryptographic and hash algorithm based on chaotic control character," Communications in Nonlinear Science and Numerical Simulation, vol. 14, no. 11, pp. 3889-3900, 2009. 
26. S. Deng, Y. Li, and D. Xiao, "Analysis and improvement of a chaos-based hash function construction," Communications in Nonlinear Science and Numerical Simulation, vol. 15, no. 5, pp. 1338-1347, 2010.

27. H. Yang, K.-W. Wong, X. Liao, Y. Wang, and D. Yang, "One-way hash function construction based on chaotic map network," Chaos, Solitons \& Fractals, vol. 41, no. 5, pp. 2566-2574, 2009.

28. D. Xiao, X. Liao, and Y. Wang, "Improving the security of a parallel keyed hash function based on chaotic maps," Physics Letters A, vol. 373, no. 47, pp. 4346-4353, 2009.

29. Y. Li, S. Deng, and D. Xiao, "A novel hash algorithm construction based on chaotic neural network," Neural Computing and Applications, vol. 20, no. 1, pp. 133-141, 2011.

30. Y. Li, D. Xiao, S. Deng, Q. Han, and G. Zhou, "Parallel hash function construction based on chaotic maps with changeable parameters," Neural Computing and Applications, vol. 20, no. 8, pp. 1305-1312, 2011.

31. Y. Li, D. Xiao, S. Deng, and G. Zhou, "Improvement and performance analysis of a novel hash function based on chaotic neural network," Neural Computing and Applications, vol. 22, no. 2, pp. 391-402, 2013.

32. B. He, P. Lei, Q. Pu, and Z. Liu, "A method for designing hash function based on chaotic neural network," in International Workshop on Cloud Computing and Information Security (CCIS), 2013.

33. Z. Huang, "A more secure parallel keyed hash function based on chaotic neural network," Communications in Nonlinear Science and Numerical Simulation, vol. 16, no. 8, pp. 3245$3256,2011$.

34. N. Jiteurtragool, P. Ketthong, C. Wannaboon, and W. San-Um, "A topologically simple keyed hash function based on circular chaotic sinusoidal map network," in Advanced Communication Technology (ICACT), 2013 15th International Conference on, pp. 1089-1094, IEEE, 2013.

35. J. S. Teh, A. Samsudin, and A. Akhavan, "Parallel chaotic hash function based on the shuffle-exchange network," Nonlinear Dynamics, vol. 81, no. 3, pp. 1067-1079, 2015.

36. N. Abdoun, S. El Assad, M. A. Taha, R. Assaf, O. Deforges, and M. Khalil, "Hash function based on efficient chaotic neural network," in International Conference on Internet Technology and Secured Transactions, pp. 32-37, 2015.

37. N. Abdoun, S. El Assad, M. A. Taha, R. Assaf, O. Déforges, and M. Khalil, "Secure hash algorithm based on efficient chaotic neural network," in The 11th International Conference on Communications, p. comm2016, 2016.

38. M. A. Chenaghlu, S. Jamali, and N. N. Khasmakhi, "A novel keyed parallel hashing scheme based on a new chaotic system," Chaos, Solitons \& Fractals, vol. 87, pp. 216-225, 2016.

39. A. Akhavan, A. Samsudin, and A. Akhshani, "A novel parallel hash function based on 3d chaotic map," EURASIP Journal on Advances in Signal Processing, vol. 2013, no. 1, p. $126,2013$.

40. M. Nouri, A. Khezeli, A. Ramezani, and A. Ebrahimi, "A dynamic chaotic hash function based upon circle chord methods," in Telecommunications (IST), 2012 Sixth International Symposium on, pp. 1044-1049, IEEE, 2012.

41. R. Guesmi, M. Farah, A. Kachouri, and M. Samet, "A novel chaos-based image encryption using dna sequence operation and secure hash algorithm sha-2," Nonlinear Dynamics, vol. 83, no. 3, pp. 1123-1136, 2016.

42. D. Xiao, X. Liao, and S. Deng, "Parallel keyed hash function construction based on chaotic maps," Physics Letters A, vol. 372, no. 26, pp. 4682-4688, 2008.

43. S. El Assad and H. Noura, "Generator of chaotic sequences and corresponding generating system," July 15 2014. US Patent 8,781,116.

44. S. El Assad, "Chaos based information hiding and security," in Internet Technology And Secured Transactions, 2012 International Conference for, pp. 67-72, IEEE, 2012.

45. W. Stallings, Cryptography and Network Security: Principles and Practice, International Edition: Principles and Practice. Pearson Higher Ed, 2014.

46. A. J. Menezes, P. C. Van Oorschot, and S. A. Vanstone, Handbook of applied cryptography. CRC press, 1996.

47. C. Liu, H. Ling, F. Zou, Y. Wang, H. Feng, and L. Yan, "Local and global structure preserving hashing for fast digital fingerprint tracing," Multimedia Tools and Applications, vol. 74, no. 18, pp. 8003-8023, 2015.

48. S.-H. Lee, W.-J. Hwang, and K.-R. Kwon, "Polyline curvatures based robust vector data hashing," Multimedia tools and applications, vol. 73, no. 3, pp. 1913-1942, 2014. 
49. B.-K. Kim, S.-J. Oh, S.-B. Jang, and Y.-W. Ko, "File similarity evaluation scheme for multimedia data using partial hash information," Multimedia Tools and Applications, vol. 76, no. 19, pp. 19649-19663, 2017.

50. M. Bellare, R. Canetti, and H. Krawczyk, "Keying hash functions for message authentication," in Annual International Cryptology Conference, pp. 1-15, Springer, 1996.

51. B. Denton and R. Adhami, "Modern hash function construction,"

52. R. C. Merkle, R. Charles, et al., "Secrecy, authentication, and public key systems," 1979.

53. I. B. Damgård, "A design principle for hash functions," in Conference on the Theory and Application of Cryptology, pp. 416-427, Springer, 1989.

54. S. Lucks, "Design principles for iterated hash functions.," IACR Cryptology ePrint Archive, vol. 2004, p. 253, 2004.

55. M. Nandi and S. Paul, "Speeding up the wide-pipe: Secure and fast hashing.," in Indocrypt, vol. 6498, pp. 144-162, Springer, 2010.

56. O. Dunkelman and E. Biham, "A framework for iterative hash functions: Haifa," in 2nd NIST Cryptographich Hash Workshop, vol. 22, 2006.

57. G. Bertoni, J. Daemen, M. Peeters, and G. Van Assche, "Sponge functions," in ECRYPT hash workshop, vol. 2007, 2007.

58. R. Rivest, "The md5 message-digest algorithm," 1992.

59. F. PUB, "Secure hash standard," Public Law, vol. 100, p. 235, 1995.

60. S. M. Matyas, "Generating strong one-way functions with cryptographic algorithm," IBM Technical Disclosure Bulletin, vol. 27, pp. 5658-5959, 1985.

61. T. Bartkewitz, "Building hash functions from block ciphers, their security and implementation properties," Ruhr-University Bochum, 2009.

62. B. O. Brachtl, D. Coppersmith, M. M. Hyden, S. M. Matyas Jr, C. H. Meyer, J. Oseas, S. Pilpel, and M. Schilling, "Data authentication using modification detection codes based on a public one way encryption function," Mar. 13 1990. US Patent 4,908,861.

63. S. Miyaguchi, M. Iwata, and K. Ohta, "New 128-bit hash function," in Proc. 4th International Joint Workshop on Computer Communications, Tokyo, Japan, pp. 279-288, 1989.

64. B. Preneel, R. Govaerts, and J. Vandewalle, "Hash functions based on block ciphers: A synthetic approach.," in Crypto, vol. 93, pp. 368-378, Springer, 1993.

65. S. Miyaguchi, K. Ohta, and M. Iwata, "Confirmation that some hash functions are not collision free," in Workshop on the Theory and Application of of Cryptographic Techniques, pp. 326-343, Springer, 1990.

66. B. Prencel, A. Bosselaers, R. Govaerts, and J. Vandewalle, "Collision-free hashfunctions based on blockcipher algorithms," in Security Technology, 1989. Proceedings. 1989 International Carnahan Conference on, pp. 203-210, IEEE, 1989.

67. K. Desnos, S. El Assad, A. Arlicot, M. Pelcat, and D. Menard, "Efficient multicore implementation of an advanced generator of discrete chaotic sequences," in Internet Technology and Secured Transactions (ICITST), 2014 9th International Conference for, pp. 31-36, IEEE, 2014

68. B. Preneel, Analysis and design of cryptographic hash functions. PhD thesis, Katholieke Universiteit te Leuven, 1993.

69. H. Feistel, "Cryptography and computer privacy," Scienfitic American, vol. 228, pp. 15-23, 1973.

70. I. Mironov et al., "Hash functions: Theory, attacks, and applications," Microsoft Research, Silicon Valley Campus. Noviembre de, 2005.

71. K. Aoki and Y. Sasaki, "Preimage attacks on one-block md4, 63-step md5 and more," in International Workshop on Selected Areas in Cryptography, pp. 103-119, Springer, 2008.

72. P. Flajolet, D. Gardy, and L. Thimonier, "Birthday paradox, coupon collectors, caching algorithms and self-organizing search," Discrete Applied Mathematics, vol. 39, no. 3, pp. 207-229, 1992.

73. G. Yuval, "How to swindle rabin," Cryptologia, vol. 3, no. 3, pp. 187-191, 1979.

74. S. Bakhtiari, R. Safavi-Naini, J. Pieprzyk, et al., "Cryptographic hash functions: A survey," Centre for Computer Security Research, Department of Computer Science, University of Wollongong, Australie, 1995.

75. B. Preneel and P. van Oorschot, "On the security of two mac algorithms," in Advances in CryptologyEUROCRYPT96, pp. 19-32, Springer, 1996.

76. "Hash length extension attacks - java code geeks - 2017." https://www.javacodegeeks . com/2012/07/hash-length-extension-attacks.html. (Accessed on 07/11/2017). 
77. "Md5 length extension attack revisited — v's inner peace." https://web.archive. org/web/20141029080820/http://vudang.com/2012/03/md5-length-extension-attack/. (Accessed on 07/11/2017).

78. "Stop using unsafe keyed hashes, use hmac — rdist." https://rdist.root.org/2009/10/ 29/stop-using-unsafe-keyed-hashes-use-hmac/. (Accessed on 07/11/2017).

79. L. Wei, C. Rechberger, J. Guo, H. Wu, H. Wang, and S. Ling, "Improved meet-in-themiddle cryptanalysis of ktantan (poster)," in Australasian Conference on Information Security and Privacy, pp. 433-438, Springer, 2011.

80. K. Aoki and Y. Sasaki, "Meet-in-the-middle preimage attacks against reduced sha-0 and sha-1," in Advances in Cryptology-CRYPTO 2009, pp. 70-89, Springer, 2009.

81. Y. Sasaki and K. Aoki, "Finding preimages in full md5 faster than exhaustive search.," in EUROCRYPT, vol. 5479, pp. 134-152, Springer, 2009.

82. K. Aoki, J. Guo, K. Matusiewicz, Y. Sasaki, and L. Wang, "Preimages for step-reduced sha-2.," in ASIACRYPT, vol. 5912, pp. 578-597, Springer, 2009.

83. J. Guo, S. Ling, C. Rechberger, and H. Wang, "Advanced meet-in-the-middle preimage attacks: first results on full tiger, and improved results on md4 and sha-2.," in ASIACRYPT, vol. 6477, pp. 56-75, Springer, 2010.

84. D. Hong, B. Koo, and Y. Sasaki, "Improved preimage attack for 68-step has-160.," in ICISC, vol. 5984, pp. 332-348, Springer, 2009.

85. Y. Sasaki and K. Aoki, "Preimage attacks on 3, 4, and 5-pass haval," in International Conference on the Theory and Application of Cryptology and Information Security, pp. 253271 , Springer, 2008.

86. A. Joux, "Multicollisions in iterated hash functions. application to cascaded constructions," in Annual International Cryptology Conference, pp. 306-316, Springer, 2004.

87. J. Kelsey and B. Schneier, "Second preimages on n-bit hash functions for much less than 2 n work," in Annual International Conference on the Theory and Applications of Cryptographic Techniques, pp. 474-490, Springer, 2005.

88. J. Kelsey and T. Kohno, "Herding hash functions and the nostradamus attack," in Annual International Conference on the Theory and Applications of Cryptographic Techniques, pp. 183-200, Springer, 2006.

89. L. Yu-Ling and D. Ming-Hui, "One-way hash function construction based on the spatiotemporal chaotic system," Chinese Physics B, vol. 21, no. 6, p. 060503, 2012.

90. D. Xiao, F. Y. Shih, and X. Liao, "A chaos-based hash function with both modification detection and localization capabilities," Communications in Nonlinear Science and Numerical Simulation, vol. 15, no. 9, pp. 2254-2261, 2010.

91. Y. Li, D. Xiao, H. Li, and S. Deng, "Parallel chaotic hash function construction based on cellular neural network," Neural Computing and Applications, vol. 21, no. 7, pp. 15631573, 2012.

92. Y. Li, D. Xiao, and S. Deng, "Keyed hash function based on a dynamic lookup table of functions," Information Sciences, vol. 214, pp. 56-75, 2012.

93. H. Ren, Y. Wang, Q. Xie, and H. Yang, "A novel method for one-way hash function construction based on spatiotemporal chaos," Chaos, Solitons \& Fractals, vol. 42, no. 4, pp. 2014-2022, 2009.

94. X.-F. Guo and J.-S. Zhang, "Keyed one-way hash function construction based on the chaotic dynamic s-box," 2006.

95. H. Yu, Y.-f. Lu, X. Yang, and Z.-l. Zhu, "One-way hash function construction based on chaotic coupled map network," in Chaos-Fractals Theories and Applications (IWCFTA), 2011 Fourth International Workshop on, pp. 193-197, IEEE, 2011.

96. H. Zhang, X.-F. Wang, Z.-H. Li, and D.-H. Liu, "One way hash function construction based on spatiotemporal chaos," 2005. 